\title{
The role of unequal diffusivities in ignition and extinction fronts in strained mixing layers J Daou and A Liñán
}

\begin{abstract}
We have studied flame propagation in a strained mixing layer formed between a fuel stream and an oxidizer stream, which can have different initial temperatures. Allowing the Lewis numbers to deviate from unity, the problem is first formulated within the framework of a thermo-diffusive model and a single irreversible reaction. A compact formulation is then derived in the limit of large activation energy, and solved analytically for high values of the Damköhler number. Simple expressions describing the flame shape and its propagation velocity are obtained. In particular, it is found that the Lewis numbers affect the propagation of the triple flame in a way similar to that obtained in the studies of stretched premixed flames. For example, the flame curvature determined by the transverse enthalpy gradients in the frozen mixing layer leads to flame-front velocities which grow with decreasing values of the Lewis numbers.

The analytical results are complemented by a numerical study which focuses on preferentialdiffusion effects on triple flames. The results cover, for different values of the fuel Lewis number, a wide range of values of the Damköhler number leading to propagation speeds which vary from positive values down to large negative values.
\end{abstract}

\section{Introduction}

Flame propagation in inhomogeneous mixtures occurs in most practical situations. For example, spatial non-uniformities in the enthalpy of the reactants are frequently encountered in unpremixed-combustion devices. Even when such non-uniformities are weak, their impact on the initiation process and the dynamics of burning is generally important. This is due to the typical large activation energies of the chemical reactions encountered in combustion, which make their rates very sensitive to the surrounding conditions. In many instances, composition and temperature inhomogeneities are essentially transverse to mixing layers, along which flames can propagate, as in lifted jet diffusion flames. Because the combustible mixture varies from lean to rich across a mixing layer, triple flames, consisting of two premixed branches and a trailing diffusion flame, are expected. Therefore, they have been the subject of a number of experimental, analytical and numerical studies [1-6].

The main purpose of the present investigation is to determine how the propagation of the triple flame is influenced by transverse enthalpy gradients in the fresh mixture and by differential diffusion. We shall select for definiteness the strained mixing layer configuration as a frame for the investigation, and adopt additionally the constant-density approximation [7] to make the analytical description tractable. The configuration of the study is sketched 


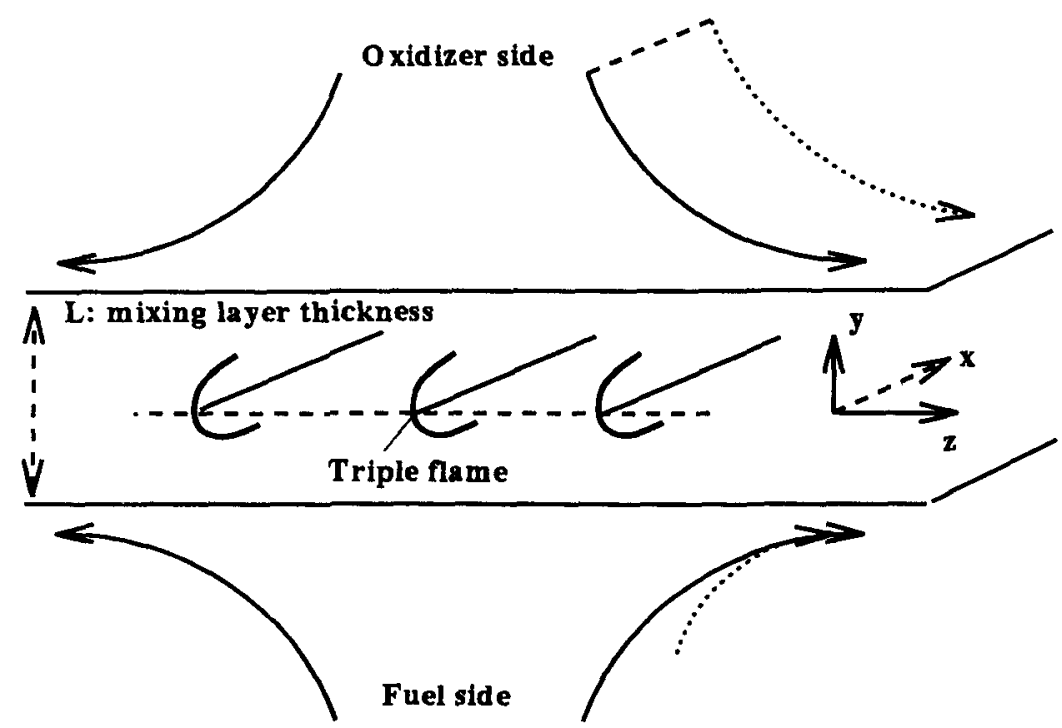

Figure 1. The strained mixing layer configuration. The fuel stream has temperature $T_{\mathrm{F}}$, a fuel mass fraction $y_{\mathrm{F}, \mathrm{F}}$ and contains no oxidizer. The oxidizer stream has temperature $T_{\mathrm{O}}$, an oxidizer mass fraction $y_{0.0}$ and contains no fuel. The density being assumed constant, the velocity field considered is a two-dimensional stagnation-point-type flow with components $v_{y}=-a Y$ and $v_{z}=a Z$ on the $y$-axis and $z$-axis, respectively. We shall study herein flame propagation along the mixing layer in the $x$-direction.

in figure 1, where useful notations are introduced. The velocity field considered is a twodimensional stagnation-point-type flow with components $v_{y}=-a Y$ and $v_{z}=a Z$ on the $y$-axis and $z$-axis, respectively, where $a$ is the strain rate.

We shall examine steady flame propagation along the mixing layer in the $x$-direction, described by similarity solutions (i.e. temperature and composition fields) which are independent of the $z$-coordinate and of time if we use a frame of reference attached to the flame front. In such a frame, the velocity field $v$ has an additional $x$-component, $U$, the flame-front velocity, which will be an eigenvalue of the problem, hence $v=(U,-a Y, a Z)$. The triple flames which we thus analyse will correspond, if $U>0$, to ignition fronts, which extend diffusion flames to the frozen regions of the mixing layer, or, if $U<0$, to extinction fronts. Our main concern will be the determination of the flame shape and the front velocity $U$.

The paper is organized into three parts. The first part is dedicated to a general formulation of the problem, within the framework of a constant-density model and a single Arrhenius reaction. Then, in the limit of large activation energy, a compact formulation is derived and solved analytically for large values of the Damköhler number. Finally, numerical results covering a wide range of the Damköhler number are presented, which focus on preferential diffusion effects on triple flames.

\section{General formulation}

The problem addressed herein is the steady propagation of a flame in a strained mixing layer along the $x$-axis as sketched in figure 1. For the sake of simplicity, the following 
assumptions are made. The density $\rho$, the thermal conductivity $\lambda$, the heat capacity $c_{p}$ and the individual species diffusion coefficients are assumed to be constant. The combustion is represented by a single irreversible one-step reaction of the form

$$
\mathrm{F}+s \mathrm{Ox} \rightarrow \mathrm{P}+q
$$

where $\mathrm{F}$ denotes the fuel, $\mathrm{Ox}$ the oxidizer and $\mathrm{P}$ the products. The quantity $s$ denotes the mass of oxidizer consumed and $q$ the heat released, both per unit mass of fuel. The combustion rate, $\omega$, defined as the mass of fuel consumed per unit volume and unit time, is assumed to follow an Arrhenius law of the form

$$
\omega=B \rho^{2} Y_{\mathrm{F}} Y_{\mathrm{O}} \exp (-E / R T)
$$

where $\mathrm{B}, Y_{\mathrm{F}}, Y_{\mathrm{O}}$ and $E / R$ represent, respectively, the (constant) pre-exponential factor, mass fraction of fuel, mass fraction of oxidizer and the activation temperature.

For the existence of a steady flame front propagating the reaction in the mixing layer, the activation energy of the reaction must be large enough so that, for strain rates between ignition and extinction values, there are three steady modes of combustion independent of $X$ in the mixing layer, corresponding to the one-dimensional strained diffusion flame. The front joins the upper, strongly burning, and lower, weakly burning modes.

The governing equations are

$$
\begin{aligned}
& U \frac{\partial Y_{\mathrm{F}}}{\partial X}=D_{\mathrm{F}}\left(\frac{\partial^{2} Y_{\mathrm{F}}}{\partial X^{2}}+\frac{\partial^{2} Y_{\mathrm{F}}}{\partial Y^{2}}\right)-\frac{\omega}{\rho}+a Y \frac{\partial Y_{\mathrm{F}}}{\partial Y} \\
& U \frac{\partial Y_{\mathrm{O}}}{\partial X}=D_{\mathrm{O}}\left(\frac{\partial^{2} Y_{\mathrm{O}}}{\partial X^{2}}+\frac{\partial^{2} Y_{\mathrm{O}}}{\partial Y^{2}}\right)-s \frac{\omega}{\rho}+a Y \frac{\partial Y_{\mathrm{O}}}{\partial Y} \\
& U \frac{\partial T}{\partial X}=D_{T}\left(\frac{\partial^{2} T}{\partial X^{2}}+\frac{\partial^{2} T}{\partial Y^{2}}\right)+\frac{q}{c_{p}} \frac{\omega}{\rho}+a Y \frac{\partial T}{\partial Y} .
\end{aligned}
$$

Here $D_{\mathrm{F}}, D_{\mathrm{O}}$ and $D_{\mathrm{T}}$ denote the diffusion coefficients for the fuel, the oxidizer and for heat, respectively.

The conditions at $X=-\infty$ correspond to the lower, weakly burning solution independent of $X$ which, if the activation energy is large enough, is very close to the frozen solution

$$
\begin{aligned}
& Y_{\mathrm{F} . \mathrm{fr}}=\frac{Y_{\mathrm{F} . \mathrm{F}}}{2}\left[1-\operatorname{erf}\left(\frac{Y}{\sqrt{2 D_{\mathrm{F}} / a}}\right)\right] \\
& Y_{\mathrm{O} . \mathrm{fr}}=\frac{Y_{\mathrm{O} . \mathrm{O}}}{2}\left[1+\operatorname{erf}\left(\frac{Y}{\sqrt{2 \bar{D}_{\mathrm{O} / a}}}\right)\right] \\
& T_{\mathrm{fr}}=\frac{T_{\mathrm{F}}+T_{\mathrm{O}}}{2}+\frac{T_{\mathrm{O}}-T_{\mathrm{F}}}{2} \operatorname{erf}\left(\frac{Y}{\sqrt{2 D_{\mathrm{T}} / a}}\right)
\end{aligned}
$$

where the subscripts $F$ and $O$ refer to the values of the concentrations and temperature on the fuel side and oxidizer side, respectively. These were used in the boundary conditions (at $|Y| \rightarrow \infty, X \rightarrow-\infty$ ) to obtain (2), and must be used at $|Y| \rightarrow \infty$ for all $X$. The subscript ' $f r$ ' is added as a reminder that the values correspond to the fresh (or frozen) side of the mixing layer. Downstream, for $X \rightarrow \infty$, the solution again becomes independent of $X$, corresponding to the one-dimensional strong-burning solution of the strained diffusion flame.

Since the flame-front region is expected to be centred around the stoichiometric surface, we shall use the scaled quantities

$$
y_{\mathrm{F}}=\frac{Y_{\mathrm{F}}}{Y_{\mathrm{F} . \mathrm{st}}} \quad y_{\mathrm{O}}=\frac{Y_{\mathrm{O}}}{Y_{\mathrm{O} . \mathrm{st}}} \quad \text { and } \quad \theta=\frac{T-T_{\mathrm{st}}}{T_{\mathrm{ad}}-T_{\mathrm{st}}} .
$$


Here the subscript 'st' indicates values at $\left(X=-\infty, Y=Y_{\mathrm{st}}\right)$, where the stoichiometric condition $Y_{\mathrm{O} . \mathrm{fr}}=s Y_{\mathrm{F} . \mathrm{fr}}$ holds and $T_{\mathrm{ad}} \equiv T_{\mathrm{st}}+q Y_{\mathrm{F} \text {.st }} / c_{\mathrm{p}}$ is the corresponding adiabatic flame temperature. $Y_{\mathrm{st}}$, the location of the upstream stoichiometric surface, is given by

$$
S \operatorname{erf}\left(\frac{Y_{\mathrm{st}}}{\sqrt{2 D_{\mathrm{F}} / a}}\right)+\operatorname{erf}\left(\frac{Y_{\mathrm{st}}}{\sqrt{2 D_{\mathrm{O}} / a}}\right)=S-1
$$

where $S \equiv s Y_{\text {F.F }} / Y_{\text {O.O }}$

To describe the problem in a non-dimensional form, we shall select as unit speed the laminar burning speed of a stoichiometric planar flame $\dagger, S_{\mathrm{L}}^{0}$. As unit length, we shall select $L / \beta$, the expected characteristic value of the radius of curvature of the flame front; here $L=\sqrt{2 D_{\mathrm{T}} / a}$ is the thickness of the mixing layer and $\beta \equiv E\left(T_{\mathrm{ad}}-T_{\mathrm{st}}\right) / R T_{\mathrm{ad}}^{2}$ the Zeldovich number. The flame-front velocity (measured with $S_{\mathrm{L}}^{0}$ ), may be expected to deviate from one by a factor of order unity when $L / \beta$ is of the order of the laminar flame thickness $l_{\mathrm{F} 1}^{0} \equiv \lambda /\left(\rho c_{\mathrm{p}} S_{\mathrm{L}}^{0}\right)$. We shall begin here by giving a general formulation, in which

$$
\epsilon \equiv \frac{l_{\mathrm{F} 1}^{0}}{L / \beta}
$$

will be an important parameter. This formulation will be used in the numerical analysis described in section 6. Additionally, it will be simplified below into a more compact form, valid in the limit $\beta \rightarrow \infty$ with $\epsilon \sim 1$, which will be solved analytically in the cases where the radius of curvature is large compared with the laminar flame thickness $l_{\mathrm{Fl}}^{0}$ (i.e. $\epsilon \ll 1$ ).

In terms of the coordinates $y \equiv \beta\left(Y-Y_{\mathrm{st}}\right) / L$ and $x \equiv \beta X / L$ the non-dimensional governing equations are

$$
\begin{aligned}
& U \frac{\partial y_{\mathrm{F}}}{\partial x}=\frac{\epsilon}{L e_{\mathrm{F}}}\left(\frac{\partial^{2} y_{\mathrm{F}}}{\partial x^{2}}+\frac{\partial^{2} y_{\mathrm{F}}}{\partial y^{2}}\right)-\epsilon^{-1} \tilde{\omega}+\frac{2 \epsilon}{\beta}\left(\eta_{s}+\frac{y}{\beta}\right) \frac{\partial y_{\mathrm{F}}}{\partial y} \\
& U \frac{\partial y_{\mathrm{O}}}{\partial x}=\frac{\epsilon}{L e_{\mathrm{O}}}\left(\frac{\partial^{2} y_{\mathrm{O}}}{\partial x^{2}}+\frac{\partial^{2} y_{\mathrm{O}}}{\partial y^{2}}\right)-\epsilon^{-1} \tilde{\omega}+\frac{2 \epsilon}{\beta}\left(\eta_{s}+\frac{y}{\beta}\right) \frac{\partial y_{\mathrm{O}}}{\partial y} \\
& U \frac{\partial \theta}{\partial x}=\epsilon\left(\frac{\partial^{2} \theta}{\partial x^{2}}+\frac{\partial^{2} \theta}{\partial y^{2}}\right)+\epsilon^{-1} \tilde{\omega}+\frac{2 \epsilon}{\beta}\left(\eta_{s}+\frac{y}{\beta}\right) \frac{\partial \theta}{\partial y}
\end{aligned}
$$

to be solved with the boundary conditions (2), which in terms of $y$ take the form

$$
\begin{aligned}
& y_{\mathrm{F}}=\frac{1-\operatorname{erf}\left(\left(\eta_{\mathrm{s}}+y / \beta\right) \sqrt{L e_{\mathrm{F}}}\right)}{1-\operatorname{erf}\left(\eta_{\mathrm{s}} \sqrt{L e_{\mathrm{F}}}\right)} \\
& y_{\mathrm{O}}=\frac{1+\operatorname{erf}\left(\left(\eta_{\mathrm{s}}+y / \beta\right) \sqrt{L e_{\mathrm{O}}}\right)}{1+\operatorname{erf}\left(\eta_{\mathrm{s}} \sqrt{L e_{\mathrm{O}}}\right)} \\
& \theta=\Upsilon \frac{\operatorname{erf}\left(\eta_{\mathrm{s}}+y / \beta\right)-\operatorname{erf}\left(\eta_{\mathrm{s}}\right)}{1-\operatorname{erf}\left(\eta_{\mathrm{s}} \sqrt{L e_{\mathrm{F}}}\right)} \quad \text { as } x \rightarrow-\infty \text { or }|y| \rightarrow \infty .
\end{aligned}
$$

Here $L e_{\mathrm{F}} \equiv D_{\mathrm{T}} / D_{\mathrm{F}}$ and $L e_{\mathrm{O}} \equiv D_{\mathrm{T}} / D_{\mathrm{O}}$ are the Lewis numbers of the fuel and of the oxidizer, respectively, and $\eta_{s}$ and $\Upsilon$ are two non-dimensional parameters characterizing the location of the stoichiometric surface and the transverse temperature gradient in the frozen mixturef:

$$
\eta_{\mathrm{s}} \equiv \frac{Y_{\mathrm{st}}}{\sqrt{2 D_{\mathrm{T}} / a}} \quad \text { and } \quad \Upsilon \equiv \frac{T_{\mathrm{O}}-T_{\mathrm{F}}}{q Y_{\mathrm{F}, \mathrm{F}} / c_{\mathrm{p}}} .
$$

$\dagger$ We have taken $S_{\mathrm{L}}^{0}=\left(4 \beta^{-3} Y_{\mathrm{O}, \mathrm{st}}\left(\lambda / c_{\mathrm{p}}\right) B \exp \left(-E / R T_{\mathrm{ad}}\right)\right)^{1 / 2}$, which is the first approximation for $\beta \gg 1$ of the burning speed of a planar flame corresponding to the stoichiometric conditions prevailing at $\left(X=-\infty, Y=Y_{\mathrm{st}}\right)$ and for unity Lewis numbers.

$\ddagger$ The parameter $\eta_{\mathrm{s}}$, used in this study instead of the stoichiometric coefficient $S$ defined above, is of course related to $S$ by $S \operatorname{erf}\left(\eta_{s} \sqrt{L e_{\mathrm{F}}}\right)+\operatorname{erf}\left(\eta_{\mathrm{s}} \sqrt{L e_{\mathrm{O}}}\right)=S-1$ according to (3). 
Downstream, the solutions correspond to the strongly burning solution of the planar diffusion flame, independent of $x$. Thus, we shall impose as boundary conditions

$$
\frac{\partial y_{\mathrm{F}}}{\partial x}=\frac{\partial y_{\mathrm{O}}}{\partial x}=\frac{\partial \theta}{\partial x}=0 \quad \text { for } \quad x \rightarrow \infty .
$$

The non-dimensional reaction rate $\widetilde{\omega}$ is given by

$$
\tilde{\omega}=\frac{\beta^{3}}{4} y_{\mathrm{F}} y_{\mathrm{O}} \exp \left(\frac{\beta(\theta-1)}{1+\alpha_{\mathrm{h}}(\theta-1)}\right)
$$

where $\alpha_{\mathrm{h}} \equiv\left(T_{\mathrm{ad}}-T_{\mathrm{st}}\right) / T_{\mathrm{ad}}$ is a heat-release parameter.

The solution to the above problem $\dagger$, equations (4), (5) and (7), will provide the flamefront velocity $U$ in terms of $\epsilon, L e_{\mathrm{F}}, L e_{\mathrm{O}}, \eta_{\mathrm{s}}$ and $\Upsilon$ (in addition to $\beta$ and $\alpha_{\mathrm{h}}$ ). The results of the numerical analysis of the cases where $\epsilon$ is not necessarily small is given in section 6 . Specifically, we shall describe there the influence of two parameters, $\epsilon$ and $L e_{\mathrm{F}}$, on triple flames. The other parameters will be assigned fixed values, and variations in their values will not be considered in the numerical study. We shall, however, describe in some detail the limiting case where $\epsilon \ll 1$, corresponding to large values of the Damköhler numberł. To this end, we shall carry out below an asymptotic analysis, where a reformulation of the problem is obtained in the distinguished limit $\beta \rightarrow \infty$ with $\epsilon \sim 1$, and solved analytically for $\epsilon \ll 1$.

Remark. At this point, the reader who is already familiar with the problem formulation and notation so far, and who is not interested in the details of the analysis and the derivation of the results, may move directly to section 7 . For his convenience, a short summary of the main findings is given there.

\section{Orders of magnitude of the scales for the different propagation regimes}

The problem of triple-flame propagation, as formulated in equations (4), (5) and (7), is expected to have different regimes including positive and negative flame speeds. These regimes have been studied in detail by Dold and Hartley (see, e.g., $[2,3])$ for unity Lewis numbers. In this section we shall simply make a few qualitative remarks so as to make explicit the relevant orders of magnitude in the present notation. For simplicity, we shall also consider the Lewis numbers to be unity in this qualitative discussion.

The main length scales of the problem under consideration are $L, L / \beta, l_{\mathrm{F}}^{0}$ and $l_{\mathrm{h}}$, respectively the mixing layer thickness, the typical radius of curvature of the leading premixed front $\S$, the thickness of a stoichiometric planar flame and the preheat thickness of the curved premixed front. The discussion will be in terms of the parameter $\epsilon \equiv l_{\mathrm{Fl}}^{0} /(L / \beta)$. The different regimes expected for large values of $\beta$ are:

† To a large extent, the results could be viewed as a generalization of those by Dold and Hartley $[2,3]$.to the non-unity Lewis numbers cases. Attempts to obtain solutions of similar problems using lumped one-dimensional approximations can be found in the literature, see [12] and references therein.

$\ddagger$ A relevant Damköhler number, $D a$, can be defined as the diffusion time across the mixing layer, $L^{2} / D_{\mathrm{T}}=2 a^{-1}$ divided by the flame transit time (over its thickness), $t_{\mathrm{L}}=\left(l_{\mathrm{Fl}}^{0}\right)^{2} / D_{\mathrm{T}}$, and hence $D a=2 \beta^{2} \epsilon^{-2}$. $D a$ is also equal to $2 / K a$, where $K a=a t_{\mathrm{L}}$ is the non-dimensional strain or Karlovitz number.

$\S$ Obviously, $L / \beta$ is also the transverse thickness of the region, where the reaction zones of the leading premixed front and the trailing diffusion flame lie. This region appears as a semi-infinite surface with a sharp edge situated at the leading edge of the triple flame on the mixing-layer scale, $L$. 
- $\epsilon \ll 1$. In this regimet the premixed front is quasiplanar, since it is thin, with typical thickness of order $\epsilon$ relative to its radius of curvature.

- $\epsilon \sim 1$. The preheat zone of the premixed front is of the order of its radius of curvature, $L / \beta$. Then, $U \sim 1$ so as to ensure a convective-diffusive balance in the preheat zone.

- $\epsilon \sim \beta^{1 / 2}$. This distinguished regime is obtained when the preheat length becomes of the order of the mixing layer thickness, $l_{\mathrm{h}} \sim L$. It involves propagation velocities satisfying the scaling $U \sim \beta^{-1 / 2}$, which in this regime may be negative or positive.

To justify these conclusions we first note that, for $l_{\mathrm{h}} \sim L$, it is appropriate to rescale the problem by choosing $L$ as the unit length, instead of scaling by $L / \beta$ which has led to equations (4). Thus, in terms of $x_{1}=x / \beta$ and $y_{1}=y / \beta$, equation (4c), for example, becomes

$$
\frac{\beta U}{\epsilon} \frac{\partial \theta}{\partial x_{1}}=\left(\frac{\partial^{2} \theta}{\partial x_{1}^{2}}+\frac{\partial^{2} \theta}{\partial y_{1}^{2}}\right)+2\left(\eta_{s}+y_{1}\right) \frac{\partial \theta}{\partial y_{1}}+\frac{\beta^{5}}{4 \epsilon^{2}} y_{\mathrm{F}} y_{O} \exp \left(\frac{\beta(\theta-1)}{1+\alpha_{\mathrm{h}}(\theta-1)}\right) .
$$

On the new scale, the problem separates into an outer convective-diffusive region, with typical size of order unity, and an inner diffusive-reactive region which appears as an infinitely thin semi-infinite surface with temperature equal to one in a first approximation. In the outer region, the three first terms of the preceding equation, representing longitudinal convection, diffusion and strain, respectively, are typically of the same order for distances of the leading edge of order unity, $x_{1} \sim 1$; hence, we have $\beta U / \epsilon \sim 1$. The solution of the outer problem is expected to present a square root singularity at the sharp leading edge, of the form $\ddagger \theta \sim 1-C \sqrt{r_{1}} \sin (\phi / 2)$ where $C$ depends on two parameters, namely $C=C\left(\beta U / \epsilon, \eta_{\mathrm{s}}\right)$, the first of which is $\mathrm{O}(1)$ in the regime under consideration. Hence, the temperature gradient at the reaction sheet, $r_{1} \sim \beta^{-1}$, is given by $\partial \theta / \partial r_{1} \sim \sqrt{\beta}$. Consequently, the thickness of the reaction layer of the premixed front, $\delta_{1 \mathrm{r}}$, is given by $\delta_{\mathrm{lr}} \sqrt{\beta} \sim \beta^{-1}$ or $\delta_{\mathrm{lr}} \sim \beta^{-3 / 2}$. The reactive-diffusive balance in this thin reaction layer can be written as

$$
\frac{\partial^{2} \theta}{\partial n^{2}} \sim \frac{\beta^{-1}}{\delta_{1 \mathrm{r}}^{2}} \sim \frac{\beta^{5}}{4 \epsilon^{2}}\left(\beta^{-1}\right)^{2}
$$

where $n$ denotes a coordinate normal to the reaction layer. Hence $\epsilon \sim \beta^{1 / 2}$ and $U \sim \beta^{-1 / 2}$, as advanced.

- $\epsilon \sim \beta$. A steadily propagating triple flame which, for a given $\epsilon$, connects two steady solutions of the one-dimensional strained diffusion flame, cannot of course exist except if $\epsilon$ is in the range $\left[\epsilon_{\mathrm{ign}}, \epsilon_{\mathrm{ext}}\right]$, corresponding to the existence of multiple solutions of the diffusion flame. Thus, total extinction of the triple flame will occur if $\epsilon$ exceeds $\epsilon_{\mathrm{ext}}$, given in order of magnitude by

$$
\frac{\beta^{-1}}{\delta_{\mathrm{E}}^{2}} \sim \frac{\beta^{3}}{\epsilon^{2}}\left(\beta^{-1}\right)^{2} \quad \text { and } \quad \delta_{\mathrm{E}} \sim 1
$$

or $\epsilon_{\mathrm{ext}} \sim \beta$. In this regime, and for $\epsilon<\epsilon_{\mathrm{ext}}, U$ is expected to take large negative values of order $\beta$ [2], which can also be shown by order-of-magnitude arguments similar to those given above.

$\dagger$ Of course, $\epsilon$ should be larger than a critical value, $\epsilon_{\mathrm{ign}}$, corresponding to the ignition of the one-dimensional diffusion flame.

$\ddagger r_{1}$ is the distance from the leading edge and $\phi$ is a polar angle relative to the $x$-axis, such that $\phi=0$ corresponds to the infinitely thin reaction layer. 


\section{Asymptotic analysis for large activation energy and $\epsilon \sim 1$}

In this section the distinguished limit $\beta \rightarrow \infty$, with $\epsilon \sim 1$, is considered. Then the reaction is confined to an infinitely thin reaction sheet which we shall refer to as the flame surface, and which will be given by $F(x, y)=x-f(y)=0$. Upstream, the effects of diffusion and heat conduction will reach a region where $f(y)-x$ is of order unity $\dagger$. The analysis will be restricted to the near-equidiffusion cases for which $l_{\mathrm{F}} \equiv \beta\left(L e_{\mathrm{F}}-1\right)$ and $l_{\mathrm{O}} \equiv \beta\left(L e_{\mathrm{O}}-1\right)$ are of order 1. Then, appropriate jump conditions can be derived across the reaction sheet and the problem can be reformulated in a way free from the presence of $\beta$ (see [8], for example).

We shall use a coordinate system attached to the flame

$$
\xi=x-f(y) \quad y=y
$$

so that the flame surface is located at $\xi=0$. In terms of the new coordinates the Laplacian $\Delta=\partial^{2} / \partial x^{2}+\partial^{2} / \partial y^{2}$ takes the form

$$
\Delta=\left(1+f^{\prime 2}\right) \frac{\partial^{2}}{\partial \xi^{2}}+\frac{\partial^{2}}{\partial y^{2}}-f^{\prime \prime} \frac{\partial}{\partial \xi}-2 f^{\prime} \frac{\partial}{\partial \xi \partial y} .
$$

In the limit considered, the upstream boundary conditions (5) take the linear form

$$
y_{\mathrm{F}}=1-\frac{\gamma_{\mathrm{F}}}{\beta} y \quad y_{\mathrm{O}}=1+\frac{\gamma_{\mathrm{O}}}{\beta} y \quad \theta=\frac{\gamma_{\theta}}{\beta} y
$$

if the flame-front region, $y \sim 1$, is considered and terms of order $\beta^{-2}$ neglected. Here $\gamma_{\mathrm{F}}$, $\gamma_{0}$ and $\gamma_{\theta}$ are given by

$$
\begin{aligned}
& \gamma_{\mathrm{F}}=\frac{2 \exp \left(-\eta_{\mathrm{s}}^{2}\right)}{\sqrt{\pi}\left(1-\operatorname{erf}\left(\eta_{\mathrm{s}}\right)\right)} \\
& \gamma_{0}=\frac{2 \exp \left(-\eta_{\mathrm{s}}^{2}\right)}{\sqrt{\pi}\left(1+\operatorname{erf}\left(\eta_{\mathrm{s}}\right)\right)} \\
& \gamma_{\theta}=\frac{2 \exp \left(-\eta_{\mathrm{s}}^{2}\right)}{\sqrt{\pi}\left(1-\operatorname{erf}\left(\eta_{\mathrm{s}}\right)\right)} \Upsilon .
\end{aligned}
$$

The dependent variables will be expanded in terms of $\beta^{-1}$ as

$$
\begin{aligned}
& y_{\mathrm{F}}=y_{\mathrm{F}}^{0}+\beta^{-1} y_{\mathrm{F}}^{1}+\cdots \\
& y_{\mathrm{O}}=y_{\mathrm{O}}^{0}+\beta^{-1} y_{\mathrm{O}}^{1}+\cdots \\
& \theta=\theta^{0}+\beta^{-1} \theta^{1}+\cdots
\end{aligned}
$$

Note that superscripts are used to indicate the different orders of the expansions in terms of $\beta^{-1}$. Expansions in terms of $\epsilon$ to be introduced later will be expressed by subscripts to avoid confusion. For $\beta \gg 1$ the reaction zone is thin, of extent $\xi \sim \epsilon / \beta$, so that it can be described by the diffusive-reactive balance obtained by dropping the convective terms in (4) and retaining only the first term on the right-hand side of (10).

Since the boundary conditions (11) introduce $O\left(\beta^{-1}\right)$ non-uniformities in the concentration of the reactants and their temperature, the corresponding variations in the burnt gas relative to the uniform case (that is the planar flame case) are also expected to be

$\dagger$ More precisely of order $\epsilon$. This remark is relevant since the distinguished regime under consideration extends to values of $\epsilon$ which can be either small or large compared with unity. In this latter case, $\epsilon$ must be small compared with $\beta^{1 / 2}$, as we shall comment. 
$\mathrm{O}\left(\beta^{-1}\right)$. Thus we shall write for the reaction zone and behind it for the burnt gas, i.e. for $\xi \geqslant 0$ :

$$
\theta^{0}=1 \quad y_{\mathrm{F}}^{0}=0 \quad y_{\mathrm{O}}^{0}=0 .
$$

Now defining $Z_{\mathrm{F}}$ and $Z_{\mathrm{O}}$ by

$$
Z_{\mathrm{F}} \equiv \theta+y_{\mathrm{F}} \quad Z_{\mathrm{O}} \equiv \theta+y_{\mathrm{O}}
$$

it follows from (4) and (9) that

$$
\begin{aligned}
& U \frac{\partial Z_{\mathrm{F}}}{\partial \xi}=\epsilon \Delta Z_{\mathrm{F}}-\epsilon \frac{l_{\mathrm{F}}}{\beta} \Delta y_{\mathrm{F}}+\frac{2 \epsilon}{\beta}\left(\eta_{\mathrm{s}}+\frac{y}{\beta}\right)\left[\frac{\partial Z_{\mathrm{F}}}{\partial y}-f^{\prime}(y) \frac{\partial Z_{\mathrm{F}}}{\partial \xi}\right] \\
& U \frac{\partial Z_{\mathrm{O}}}{\partial \xi}=\epsilon \Delta Z_{\mathrm{O}}-\epsilon \frac{l_{\mathrm{O}}}{\beta} \Delta y_{\mathrm{O}}+\frac{2 \epsilon}{\beta}\left(\eta_{\mathrm{s}}+\frac{y}{\beta}\right)\left[\frac{\partial Z_{\mathrm{O}}}{\partial y}-f^{\prime}(y) \frac{\partial Z_{\mathrm{O}}}{\partial \xi}\right] .
\end{aligned}
$$

The expansions of $Z_{\mathrm{F}}$ and $Z_{\mathrm{O}}$ in terms of $\beta^{-1}$ are of the form

$$
Z_{\mathrm{F}}=1+\beta^{-1} h(\xi, y)+\cdots \quad Z_{\mathrm{O}}=1+\beta^{-1} k(\xi, y)+\cdots
$$

because the upstream non-uniformities in $y_{\mathrm{F}}, y_{\mathrm{O}}$ and $\theta$ are of order $\beta^{-1}$, so that

$$
\begin{array}{ll}
\theta^{0}+y_{\mathrm{F}}^{0}=1 & \theta^{0}+y_{\mathrm{O}}^{0}=1 \\
h \equiv \theta^{1}+y_{\mathrm{F}}^{1} & k \equiv \theta^{1}+y_{\mathrm{O}}^{1}
\end{array}
$$

where (18b) defines the excess enthalpy functions $h$ and $k$ appearing in (17). Behind the reaction sheet $\xi>0$ the reaction ceases because one of the reactants has been depleted. Thus we can write for $\xi>0$ :

$$
\begin{array}{llll}
\theta^{1}=h & y_{\mathrm{F}}^{1}=0 & y_{\mathrm{O}}^{1}=k-h & \text { (fuel-lean side, } k>h \text { ) } \\
\theta^{1}=k & y_{\mathrm{O}}^{1}=0 & y_{\mathrm{F}}^{1}=h-k & \text { (fuel-rich side, } k<h \text { ). }
\end{array}
$$

Now, on using (4), (16) and (17), we obtain the governing equations for $\theta^{0}, h$ and $k$, valid at both sides of the reaction sheet $t$ :

$$
\begin{aligned}
& U \frac{\partial \theta^{0}}{\partial \xi}=\epsilon \Delta \theta^{0} \\
& U \frac{\partial h}{\partial \xi}=\epsilon \Delta h+\epsilon l_{\mathrm{F}} \Delta \theta^{0} \\
& U \frac{\partial k}{\partial \xi}=\epsilon \Delta k+\epsilon l_{\mathrm{O}} \Delta \theta^{0} .
\end{aligned}
$$

Note that the terms associated with strain (the last terms in (4) and (16)) have dropped out in the preceding equations: those are of order $\beta^{-2}$, which follows from (18a). The jump conditions resulting from integration of the quasi-planar reaction-diffusion equations are

$$
\begin{aligned}
& {\left[\theta^{0}\right]=[h]=[k]=0} \\
& {\left[\frac{\partial h}{\partial \xi}\right]=-l_{\mathrm{F}}\left[\frac{\partial \theta^{0}}{\partial \xi}\right]} \\
& {\left[\frac{\partial k}{\partial \xi}\right]=-l_{0}\left[\frac{\partial \theta^{0}}{\partial \xi}\right]} \\
& \epsilon \sqrt{1+f^{\prime 2}}\left[\frac{\partial \theta^{0}}{\partial \xi}\right]=-\left(1+\frac{1}{2}(\mu-\sigma)\right)^{1 / 2} \exp (\sigma / 2)
\end{aligned}
$$

$\dagger$ The last two equations in (20) are, in fact, also valid through the reaction sheet 
to be satisfied at $\xi=0$. Here we use the notations

$$
\begin{aligned}
& \sigma \equiv \theta_{b}^{1}=h\left(0^{+}, y\right) \quad \mu=k\left(0^{+}, y\right) \quad(k>h) \\
& \sigma \equiv \theta_{b}^{1}=k\left(0^{+}, y\right) \quad \mu=h\left(0^{+}, y\right) \quad(k<h) .
\end{aligned}
$$

The bracket applied to any quantity $\psi$ denotes the difference between the values of $\psi$ at both sides of the reaction sheet, namely, $[\psi] \equiv \psi\left(\xi=0^{+}, y\right)-\psi\left(\xi=0^{-}, y\right)$. Also the boundary conditions at $\xi=-\infty$ for $\theta^{\circ}, h$ and $k$ follow from (11), (13) and the definition of $h$ and $k$ :

$\theta^{0}=0 \quad h=\left(-\gamma_{\mathrm{F}}+\gamma_{\theta}\right) y \quad k=\left(\gamma_{\mathrm{O}}+\gamma_{\theta}\right) y \quad$ as $\quad \xi \rightarrow-\infty$.

The boundary conditions at $|y| \rightarrow \infty$ and $\xi$ finite are also given by equations (23). These are exact solutions of equations (20) and (21), if we restrict our analysis to the only cases that we consider, where $\gamma_{\theta}-\gamma_{\mathrm{F}}<0$ and $\gamma_{\theta}+\gamma_{\mathrm{O}}>0$.

At this point the problem has been reformulated, in terms of the new dependent variables $\theta^{0}, h$ and $k$. A further simplification can be obtained since it is possible to obtain $h$ and $k$ in terms of a single function $g(\xi, y)$ by the relations $\dagger$

$$
h=\left(\gamma_{\theta}-\gamma_{\mathrm{F}}\right) y+l_{\mathrm{F}}\left(g-\theta^{0}\right) \quad k=\left(\gamma_{\theta}+\gamma_{\mathrm{O}}\right) y+l_{\mathrm{O}}\left(g-\theta^{0}\right)
$$

where $g$ is continuous and has continuous gradients at the reaction sheet and is governed by

$$
U \frac{\partial g}{\partial \xi}=\epsilon \Delta g+U \frac{\partial \theta^{0}}{\partial \xi}
$$

satisfying the boundary conditions $g \rightarrow 0$ both at $\xi \rightarrow-\infty$ and $|y| \rightarrow \infty$ and $\partial g / \partial \xi=0$ for $\xi \rightarrow \infty$.

The perturbation, $\sigma$, in the premixed flame temperature can be obtained from the relations (24) and the value, $g^{P}$, of $g$ at $\xi=0$. Behind the lean and rich branches of the premixed flame front, there is also a thin diffusion-controlled reaction layer or diffusion flame located where $h=k$ or $g=g^{D}$, with $g^{D}$ being given by (24) for $h=k$ and $\theta^{0}=1$. More explicitly, if $\Delta$ is defined by

$$
\Delta=\Delta(\xi, y) \equiv\left(\gamma_{\mathrm{F}}+\gamma_{\mathrm{O}}\right) y+\left(l_{\mathrm{O}}-l_{\mathrm{F}}\right)(g-1)
$$

then the thin diffusion-controlled reaction layer is given by $\Delta=0$, a relation between $\xi$ and $y$, and the fuel-lean region behind the triple flame is given by $\Delta>0$. However, for brevity, we shall use the expression fuel-lean side to refer to conditions at the reaction sheet, $\xi=0$, thus the domain of $y$ corresponding to $\Delta(0, y)>0$, or $g=g^{P}$ and $\Delta>0$.

In summary, the limit $\beta \rightarrow \infty$ and the assumption that the Lewis numbers are close to unity allow us to replace the original conservation equations and associated boundary and jump conditions to be verified by $\theta, y_{\mathrm{F}}$ and $y_{\mathrm{O}}$ by similar expressions written in terms of $\theta^{0}, g$.

Specifically, our task now is to solve the equations

$$
\begin{aligned}
& U \frac{\partial \theta^{0}}{\partial \xi}=\epsilon \Delta \theta^{0} \\
& U \frac{\partial g}{\partial \xi}=\epsilon \Delta g+U \frac{\partial \theta^{0}}{\partial \xi}
\end{aligned}
$$

$\dagger$ Indeed, substitution of the relations $h=\left(\gamma_{H}-\gamma_{\mathrm{F}}\right) y+l_{\mathrm{F}}\left(\hat{h}-\theta^{(0)}\right)$ and $k=\left(\gamma_{H}+\gamma_{0}\right) y+l_{0}\left(\hat{k}-\theta^{0}\right)$, into the governing equations for $h$ and $k$ and their boundary and jump conditions shows that $\hat{h}$ and $\hat{k}$ are governed by the same equation and jump and boundary conditions. Thus $\hat{h}=\hat{k}=g$, where $g$ is as indicated. 
which are valid at both sides of the reaction sheet $(\xi<0$ and $\xi>0)$, subject to the boundary conditions $\theta^{0}=g=0$ at $\xi=-\infty$ and at $|y|=\infty$, and $\partial \theta^{0} / \partial \xi=\partial g / \partial \xi=0$ at $\xi=\infty$.

The jump conditions to be satisfied at $\xi=0$ are

$$
\begin{aligned}
& {\left[\theta^{0}\right]=[g]=0} \\
& {\left[\frac{\partial g}{\partial \xi}\right]=0} \\
& \epsilon \sqrt{1+f^{\prime 2}}\left[\frac{\partial \theta^{0}}{\partial \xi}\right]=-(1+b)^{1 / 2} \exp (\sigma / 2)
\end{aligned}
$$

where $\sigma$ and $b$ are given in terms of $g^{P}$, the value of $g$ at $\xi=0$, by

$$
\begin{array}{ll}
\sigma=\left(\gamma_{\theta}-\gamma_{\mathrm{F}}\right) y+l_{\mathrm{F}}\left(g^{P}-1\right) & \text { (fuel-lean side) } \\
\sigma=\left(\gamma_{\theta}+\gamma_{\mathrm{O}}\right) y+l_{\mathrm{O}}\left(g^{P}-1\right) & \text { (fuel-rich side) }
\end{array}
$$

and

$$
b=\frac{2 \gamma_{\theta}-\gamma_{\mathrm{F}}+\gamma_{\mathrm{O}}}{2} y+\frac{l_{\mathrm{F}}+l_{\mathrm{O}}}{2}\left(g^{P}-1\right)-\sigma .
$$

At this stage, a comment on the limitations of the reduced model just derived is in order. Note that the derivation of this model, for $\beta \rightarrow \infty$ with $\epsilon \sim 1$, has involved two approximations, namely a linearization of the boundary conditions (5) and a neglect of the strain terms in the governing equations (4). Clearly, the reduced model is not applicable far downstream, where a balance between transverse diffusion and strain is expected; the same balance that characterizes the underlying one-dimensional diffusion flame. This will occur for distances downstream, equal, in order of magnitude, to the fiame-front velocity multiplied by the diffusion time across the mixing layer, that is, in our non-dimensional notation, for $\nmid \xi \sim \beta^{2}$. This remark being made, the two approximations mentioned are justified as long as distances which are small compared to the mixing layer thickness, $y / \beta \ll 1$, need to be considered. This is so, as long as the preheat zone of the triple-flame leading front, $l_{\mathrm{h}}$, remains small compared with $L$ or, as we have seen in the last section, $\epsilon \ll \beta^{1 / 2}$. When $\epsilon$ takes values of order $\beta^{1 / 2}$, the results of the reduced model are expected to deviate from those of the original model. In particular, it is easily seen that no solution with zero propagation velocity, $U=0$, exists for the reduced model (unless frozen), due to the absence of the strain terms in the governing equations. Thus, no negative values of $U$ can be obtained, in the frame of the reduced model, by a continuous increase of $\epsilon$ from small to large values.

\section{The limit cases $\epsilon \ll 1$}

In the limit $\epsilon \rightarrow 0$, the flame, including its preheat zone, can be viewed as a surface of discontinuity located at $\xi=0$. For $\epsilon \ll 1$ the preheat zone upstream of the reaction sheet is a thin layer of thickness of order $\epsilon$. Outside this layer, diffusion and heat conduction can be neglected in a first approximation. For small values of $\epsilon$ we shall describe the solution in the form of expansions in $\epsilon$ of the form $f=f_{0}+\epsilon f_{1}, U=U_{0}+\epsilon U_{1}$ and similar expansions for $\theta$ and $g$ written for the different regions.

$\dagger$ More precisely, for $\xi \sim \beta^{2} / \epsilon$. 


\subsection{Outer solutions on both sides of the flame}

For $\xi<0$ and $\xi>0$ we seek outer expansions in the form

$$
\theta^{0}=\Theta_{0}+\epsilon \Theta_{1}+\cdots \quad g=G_{0}+\epsilon G_{1}+\cdots
$$

which we substitute into equations (27). We then obtain in a first approximation $U_{0} \partial \Theta_{0} / \partial \xi=U_{0} \partial G_{0} / \partial \xi=0$. Thus $\Theta_{0}$ and $G_{0}$ are independent of $\xi$ on both sides of the flame, although $\Theta_{0}$ has a jump across the flame. More precisely, when (14) and the boundary conditions at $\xi=-\infty$ are used, one finds

$$
\theta^{0}=\Theta_{0}= \begin{cases}0 & \text { for } \quad \xi<0 \\ 1 & \text { for } \quad \xi>0\end{cases}
$$

and

$$
g=G_{0}=0 \quad \text { for } \quad \xi<0 .
$$

\subsection{The structure of the flame}

We now analyse the thin region around $\xi=0$ where diffusive effects should be retained. To study this zone, we use the stretching transformation $\xi=\epsilon \zeta$. We seek expansions in the form

$$
\theta^{0}=\theta_{0}+\epsilon \theta_{1}+\cdots \quad g=g_{0}+\epsilon g_{1}+\cdots .
$$

When using these expansions with the jump conditions at the reaction sheet we obtain

$$
\begin{aligned}
& {\left[\theta_{0}\right]=\left[g_{0}\right]=0} \\
& {\left[\frac{\partial g_{0}}{\partial \xi}\right]=0} \\
& \epsilon \sqrt{1+f_{0}^{\prime 2}}\left[\frac{\partial \theta^{0}}{\partial \xi}\right]=-\left(1+b_{0}\right)^{1 / 2} \exp \left(\sigma_{0} / 2\right)
\end{aligned}
$$

and

$$
\begin{aligned}
& {\left[\theta_{1}\right]=\left[g_{1}\right]=0} \\
& {\left[\frac{\partial g_{1}}{\partial \xi}\right]=0} \\
& {\left[\frac{\partial \theta_{1}}{\partial \zeta}\right]=(A-F)\left[\frac{\partial \theta_{0}}{\partial \zeta}\right]}
\end{aligned}
$$

where $A$ and $F$ are defined by

$$
A \equiv \frac{\sigma_{1}}{2}+\frac{b_{1}}{2\left(1+b_{0}\right)} \quad F \equiv \frac{f_{0}^{\prime} f_{1}^{\prime}}{1+f_{0}^{\prime 2}} .
$$

In (35) and (36) we have introduced the expansions $\sigma=\sigma_{0}+\epsilon \sigma_{1}+\cdots$ and $b=b_{0}+\epsilon b_{1}+\cdots$, which are simply obtained from (29) once the expansion $g^{P}=g_{0}^{P}+\epsilon g_{1}^{P}+\cdots$ is determined.

Using (34) and the conservation equations (27) written in terms of $y$ and the inner variable $\zeta=\xi / \epsilon$ yields to leading order

$$
\begin{aligned}
& U_{0} \frac{\partial \theta_{0}}{\partial \zeta}-\left(1+f_{0}^{\prime 2}\right) \frac{\partial^{2} \theta_{0}}{\partial \zeta^{2}}=0 \\
& U_{0} \frac{\partial g_{0}}{\partial \zeta}-\left(1+f_{0}^{\prime 2}\right) \frac{\partial^{2} g_{0}}{\partial \zeta^{2}}=U_{0} \frac{\partial \theta_{0}}{\partial \zeta}
\end{aligned}
$$


The general solution of $(38 a)$ is $\theta_{0}=A+B \exp (\alpha \zeta)$, where

$$
\alpha \equiv \frac{U_{0}}{1+f_{0}^{\prime 2}}
$$

and $A$ and $B$ are constants to be determined at both sides of the reaction sheet. Thus for $\zeta>0$, we must have $\theta_{0}=1$, which is the bounded solution that matches the anticipated outer solution in the burnt gas (32) (second equation). For $\zeta<0$, the solution must be $\theta_{0}=\exp (\alpha \zeta)$ in order to satisfy the matching requirement with the upstream outer solution (32) (first equation) and the continuity requirement $\left[\theta_{0}\right]=0$ at $\zeta=0$. Thus we have

$$
\theta_{0}= \begin{cases}\exp (\alpha \zeta) & \text { for } \zeta<0 \\ 1 & \text { for } \zeta>0\end{cases}
$$

Similarly, $g_{0}$ will be independent of $\zeta$ for $\zeta>0$ so as to be bounded and given by

$$
g_{0}=(1-\alpha \zeta) \exp (\alpha \zeta) \quad \text { for } \quad \zeta \leqslant 0
$$

if we use the matching conditions with the upstream solution (33) and the jump condition (35b). From the definition $g^{P} \equiv g(0, y)$, it is seen that $g_{0}^{P}=1$. Also, since $g_{0}$ is continuous at $\zeta=0$, it follows that $g_{0}=1$ in the burnt gas, $\zeta>0$.

The jump condition $(35 c)$ allows us to determine the local burning speed $\dagger S_{0} \equiv$ $U_{0} /\left(1+f_{0}^{\prime 2}\right)^{1 / 2}$, a quantity that appears as the left-hand side of (35c) after using (39) and (40). The right-hand side of (35c) is known, because $\sigma_{0}$ and $b_{0}$ are now available from (29), (30) and $g_{0}^{P}=1$; namely,

$$
\begin{array}{lll}
\sigma_{0}=\left(-\gamma_{\mathrm{F}}+\gamma_{\theta}\right) y & b_{0}=\frac{\gamma_{\mathrm{F}}+\gamma_{\mathrm{O}}}{2} y & \text { (fuel-lean side) } \\
\sigma_{0}=\left(\gamma_{\mathrm{O}}+\gamma_{\theta}\right) y & b_{0}=-\frac{\gamma_{\mathrm{F}}+\gamma_{\mathrm{O}}}{2} y & \text { (fuel-rich side) }
\end{array}
$$

Consequently, the local burning speed is given by

$$
S_{0}=\frac{U_{0}}{\sqrt{1+f_{0}^{\prime 2}}}= \begin{cases}\mathrm{e}^{-\gamma \Gamma y}(1+\gamma y)^{1 / 2} & \text { for } y>0 \text { (fuel-lean) } \\ \mathrm{e}^{\gamma(1-\Gamma) y}(1-\gamma y)^{1 / 2} & \text { for } y<0 \text { (fuel-rich) }\end{cases}
$$

where the new parameters $\Gamma$ and $\gamma$ are defined by

$$
\Gamma \equiv \frac{\gamma_{\mathrm{F}}-\gamma_{\theta}}{\gamma_{\mathrm{F}}+\gamma_{\mathrm{O}}} \quad \gamma \equiv \frac{\gamma_{\mathrm{F}}+\gamma_{\mathrm{O}}}{2}
$$

and can be expressed in terms of $\eta_{\mathrm{s}}$ and $\Upsilon$ given in (6) as

$$
\gamma=\frac{2 \exp \left(-\eta_{s}^{2}\right)}{\sqrt{\pi}\left(1-\operatorname{erf}\left(\eta_{s}\right)^{2}\right)} \quad \text { and } \quad \Gamma=\frac{1+\operatorname{erf}\left(\eta_{s}\right)}{2}(1-\Upsilon)
$$

Equation (42), involving the eigenvalue $U_{0}$, determines the flame shape, $f_{0}(y)$, in a first approximation. For fixed values of $\Gamma$ and $\gamma$, equation (42) admits solutions for infinitely many values of $U_{0}$; but only for a single value of $U_{0}$, the solution will not present a cusp (i.e. a discontinuous slope) at its leading edge, as may be expected to be the case for freely propagating triple flames. This fixes the value of $U_{0}$, requiring that the derivative $f_{0}^{\prime}$ be

$\dagger$ The local burning speed $S$ is defined as the component of the fluid velocity ahead of the flame normal to the flame surface, $S=\left(U i-2(\epsilon / \beta)\left(\eta_{s}+y / \beta\right) j\right) \cdot n=U /\left(1+f^{\prime}(y)^{2}\right)^{1 / 2}+\mathrm{O}\left(\beta^{-1}\right)$, where $n=\left(i-f^{\prime}(y) j\right) /\left(1+f^{\prime}(y)\right)^{1 / 2}$ is the unit vector normal to the flame pointing to the burnt gas. 
zero at the leading edge-a criterion which has been used by Dold [2]. We thus find that the velocity of propagation of the triple flame only depends on $\Gamma$ and is given by

$$
U_{0}= \begin{cases}\frac{\mathrm{e}^{(\Gamma-1 / 2)}}{\sqrt{2 \Gamma}} & \text { for } 0<\Gamma \leqslant \frac{1}{2} \\ \frac{\mathrm{e}^{(-\Gamma+1 / 2)}}{\sqrt{2(1-\Gamma)}} & \text { for } \quad \frac{1}{2} \leqslant \Gamma<1 .\end{cases}
$$

This is also the maximum burning speed, obtained at the leading edge located (in a first approximation) at

$$
y^{*}= \begin{cases}\frac{(1-2 \Gamma)}{2 \gamma \Gamma} & 0<\Gamma \leqslant \frac{1}{2} \\ \frac{(1-2 \Gamma)}{2 \gamma(1-\Gamma)} & \frac{1}{2} \leqslant \Gamma<1\end{cases}
$$

It is easy to check that a leading edge exists only for values of $\Gamma$ strictly between 0 and 1. In view of (44), the condition $0<\Gamma<1$ is always fulfilled when $\Upsilon=0$, that is, whenever the fuel side and the oxidizer side have equal temperatures. In the general case, the condition $0<\Gamma<1$, ensuring the existence of a leading edge, can be written in terms of the parameter $\Upsilon$, and can be shown to be equivalent to the statement that a leading edge exists only if the temperature at the oxidizer side, and that at the fuel side, are less than the Burke-Schumann temperature of the diffusion flame in the mixing layert.

\subsection{Second approximation for the solution}

We have just determined the first approximation to the local burning speed, the triple-flame propagation velocity and, by using (42), the flame shape. Our aim now is to obtain the next approximation, that is the following terms in the expansions of these quantities in terms of $\epsilon$, and thus the influence of Lewis numbers on the propagation of the triple flame.

From (27) we obtain

$$
\begin{aligned}
& U_{0} \frac{\partial \theta_{1}}{\partial \zeta}-\left(1+f_{0}^{\prime 2}\right) \frac{\partial^{2} \theta_{1}}{\partial \zeta^{2}}=L\left(\theta_{0}\right)-U_{1} \frac{\partial \theta_{0}}{\partial \zeta} \\
& U_{0} \frac{\partial g_{1}}{\partial \zeta}-\left(1+f_{0}^{\prime 2}\right) \frac{\partial^{2} g_{1}}{\partial \zeta^{2}}=L\left(g_{0}\right)-U_{1} \frac{\partial g_{0}}{\partial \zeta}+U_{0} \frac{\partial \theta_{1}}{\partial \zeta}+U_{1} \frac{\partial \theta_{0}}{\partial \zeta}
\end{aligned}
$$

where $L$ is given by

$$
L \equiv 2 f_{0}^{\prime} f_{1}^{\prime} \frac{\partial^{2}}{\partial \zeta^{2}}-f_{0}^{\prime \prime} \frac{\partial}{\partial \zeta}-2 f_{0}^{\prime} \frac{\partial^{2}}{\partial y \partial \zeta}
$$

Downstream of the reaction sheet it is found that $\theta_{1}$ is zero so as to be bounded and to match (32) (second part). Similarly, $g_{1}$ is found to be independent of $\zeta$ for $\zeta \geqslant 0$. Thus

$$
\theta_{1}=0 \quad g_{1}=g_{1}^{P} \quad \text { for } \quad \zeta \geqslant 0
$$

where the constant $g_{1}^{P}$ is as yet undetermined.

$\dagger$ It is important to point out that our conclusion about the existence of a leading edge is based on a linearization about the stoichiometric line, and on the existence of a peak in $S_{0}(y)$. We shall not discuss here the non-generic case-in which the temperature of one of the feed streams is hotter than the Burke-Schumann temperature-for which $S_{0}(y)$ has no maximum. 
Integration of (47) from $\zeta=-\infty$ to $\zeta=0^{-}$yields

$$
\begin{aligned}
& \left(1+f_{0}^{\prime 2}\right)\left[\frac{\partial \theta_{1}}{\partial \zeta}\right]=I_{\theta}-U_{1} \\
& U_{0} g_{1}^{P}=I_{g}
\end{aligned}
$$

where we have introduced the notation

$$
I_{\theta}=\int_{-\infty}^{0} L\left(\theta_{0}\right) \mathrm{d} \zeta \quad \text { and } \quad I_{g}=\int_{-\infty}^{0} L\left(g_{0}\right) \mathrm{d} \zeta
$$

To obtain (50) we have used the continuity requirement (36a) to replace the values of $\theta_{1}$ and $g_{1}$ at $\zeta=0^{-}$by those at $\zeta=0^{+}$. We have also used $(36 b)$, along with the fact that the partial derivatives of $\theta_{1}$ and $g_{1}$ with respect to $\zeta$ are zero at $\zeta=-\infty$ on account of (32) and (33), and at $\zeta=0^{+}$on account of (49).

From (40) and (41), the integrals in (51) are found to be given by

$$
I_{\theta}=2 U_{0} F-f_{0}^{\prime \prime} \quad \text { and } \quad I_{g}=-f_{0}^{\prime \prime} \text {. }
$$

Then, with the aid of $(36 c)$ and use of (40), equations (50) reduce to

$$
\begin{aligned}
& U_{0} g_{1}^{P}=-f_{0}^{\prime \prime} \\
& U_{0} F-U_{1}=f_{0}^{\prime \prime}-U_{0} A
\end{aligned}
$$

involving $g_{1}^{P}$, the perturbation $U_{1}$ in front velocity and the functions $A$ and $F$ of $y$ defined in (37). At this stage we have two equations for three unknowns. However, it is possible to determine directly the perturbation in flame velocity, $U_{1}$, if we use the system of equations (52) at the leading edge of the flame, $y^{*}$, where $F=0$ because $f_{0}^{\prime}\left(y^{*}\right)=0$.

Thus we obtain

$$
\begin{aligned}
U_{1} & =-\left[1+\frac{l_{\mathrm{F}}}{2}-\frac{\left(l_{\mathrm{F}}-l_{\mathrm{O}}\right) / 4}{1+\gamma y^{*}}\right] \sqrt{2} \gamma \Gamma & & \left(0<\Gamma \leqslant \frac{1}{2}\right) \\
& =-\left[1+\frac{l_{\mathrm{O}}}{2}-\frac{\left(l_{\mathrm{O}}-l_{\mathrm{F}}\right) / 4}{1+\gamma y^{*}}\right] \sqrt{2} \gamma(1-\Gamma) & & \left(\frac{1}{2} \leqslant \Gamma<1\right) .
\end{aligned}
$$

With $U_{1}$ thus determined, we can use again the system of equations (52) for values of $y$ different from $y^{*}$ to calculate $g_{1}^{P}$ and $f_{1}^{\prime}$.

The results can be used in particular to calculate the next approximation to the normal burning speed, $S$, and the temperature along the flame front, $\theta_{\mathrm{Fl}} \equiv \theta\left(\xi=0^{+}, y\right)$.

For the former it is found that

$$
S(y)=S_{0}(y)(1-\tilde{L} \tilde{\kappa})
$$

where $S_{0}(y)$ is given by $(42), \tilde{\kappa} \equiv \epsilon S_{0}^{-1} f_{0}^{\prime \prime} / \sqrt{1+f_{0}^{\prime 2}}$ the flame curvature nondimensionalized with the local flame thickness, $D_{\mathrm{T}} / S_{0}(y)$, and $\tilde{L}$ a local Markstein length defined by

$$
\begin{aligned}
\widetilde{L}=\widetilde{L}(y) & =1+\frac{l_{\mathrm{F}}}{2}-\frac{\left(l_{\mathrm{F}}-l_{\mathrm{O}}\right) / 4}{1+\gamma y} \quad \text { at fuel-lean side } \\
& =1+\frac{l_{\mathrm{O}}}{2}-\frac{\left(l_{\mathrm{O}}-l_{\mathrm{F}}\right) / 4}{1+\gamma y} \quad \text { at fuel-rich side. }
\end{aligned}
$$

For the flame temperature the following expansion is found:

$$
\theta_{\mathrm{Fl}}= \begin{cases}1-\frac{2 \gamma \Gamma}{\beta} y-\frac{l_{\mathrm{F}}}{\beta U_{0}(\Gamma)} \epsilon f_{0}^{\prime \prime}(y) & \text { at fuel-lean side } \\ 1+\frac{2 \gamma(1-\Gamma)}{\beta} y-\frac{l_{\mathrm{O}}}{\beta U_{0}(\Gamma)} \epsilon f_{0}^{\prime \prime}(y) & \text { at fuel-rich side. }\end{cases}
$$


The significance of (55) is clear: the first term on the right-hand side, equal to one, is the flame temperature in the absence of gradients in the fresh mixture, that is the adiabatic flame temperature of a planar stoichiometric flame. The second term, linear in $y$, describes the deviation of flame temperature for an infinitely thin flame $(\epsilon=0)$, resulting from the linear deviation of mass fractions and of temperature in the fresh mixture from their values at the stoichiometric location (see the boundary conditions (11)). The third term indicates the combined effects of flame curvature and differential diffusion. It is zero for unity Lewis numbers, positive for Lewis numbers less than one, and negative otherwise in agreement with available knowledge on stretched premixed flames (see, for example, $[9,10]$ ).

We close this section by specifying the location of the stoichiometric surface behind the premixed flame $\xi>0$, or the position of the diffusion-flame tail of the triple flame, $y^{\text {st }}$. This is obtained, to first order in $\epsilon$, by setting $\Delta=0, g=g_{0}^{P}+\epsilon g_{1}^{P}$ and $y=y^{\text {st }}$ in (26). Hence

$$
y^{\mathrm{st}}=0+\frac{\left(l_{\mathrm{O}}-l_{\mathrm{F}}\right)}{2 \gamma U_{0}(\Gamma)} \epsilon f_{0}^{\prime \prime}(0)+\cdots .
$$

Thus, the position of the stoichiometric surface behind the flame is shifted from the upstream stoichiometric surface, $y=0$, if the Lewis numbers of the fuel and oxidizer are different. Since $f^{\prime \prime}$ is positive, it is clear that the shift is towards the oxidizer side if $L e_{\mathrm{O}}>L e_{\mathrm{F}}$.

\section{Numerical study}

In this section, we present the numerical results for triple-flame propagation corresponding to values of $\epsilon$ extending over a wide range, up to the extinction values of the underlying planar diffusion flame. The governing equations solved numerically and their boundary conditions are given by equations (4), (5) and (7). Those are discretized using a finitevolume method and solved with a multigrid method [11]. The computational domain extent in the transverse $(y-)$ direction is typically eight times the mixing layer thickness. The extent in the $x$-direction is typically 100 times the planar laminar flame thickness. The grid is a non-uniform rectangular grid with typically 100000 points.

The main result of the study, in its general form, would be to provide the propagation velocity $U$ in terms of $\epsilon, L e_{\mathrm{F}}, L e_{\mathrm{O}}, \eta_{\mathrm{s}}, \Upsilon, \alpha_{\mathrm{h}}$ and $\beta$. In this work, however, we shall only describe the influence of two parameters, namely $\epsilon$ and $L e_{\mathrm{F}}$.

The other parameters will have fixed values, namely $L e_{O}=1, \eta_{s}=0$ (i.e. the upstream stoichiometric surface is at the centre of the mixing layer), $\Upsilon=0$ (i.e. a zero tranverse temperature gradient), $\beta=8$ and $\alpha_{\mathrm{h}}=0.85$.

The results of our analysis will be presented in terms of $\epsilon$ and $l_{\mathrm{F}} \equiv \beta\left(L e_{\mathrm{F}}-1\right)$. Since the determination of the propagation velocity $U$ is the main purpose of the work, we shall begin by giving a synthesis in the form of a plot of $U$ versus $\epsilon$ for different values of $l_{\mathrm{F}}$. Then the influence of the (fuel-) Lewis number will be examined with more details as follows. First, we shall compare three cases corresponding to a fixed value of $\epsilon, \epsilon=0.5$, but to different values of $l_{\mathrm{F}}$. Second, we shall describe for fixed values of $l_{\mathrm{F}}$, how triple flames depend on $\epsilon$ as this parameter is varied.

Since steadily propagating triple flames are not expected if $\epsilon$ exceeds the extinction value $\epsilon_{\mathrm{ext}}$ of the planar diffusion flame, a plot of $\epsilon_{\mathrm{ext}}$ versus $l_{\mathrm{F}}$ will be useful in the discussion below. This plot, determined numerically by solving the underlying one-dimensional problem independent of $x$, is given in figure 2 . 


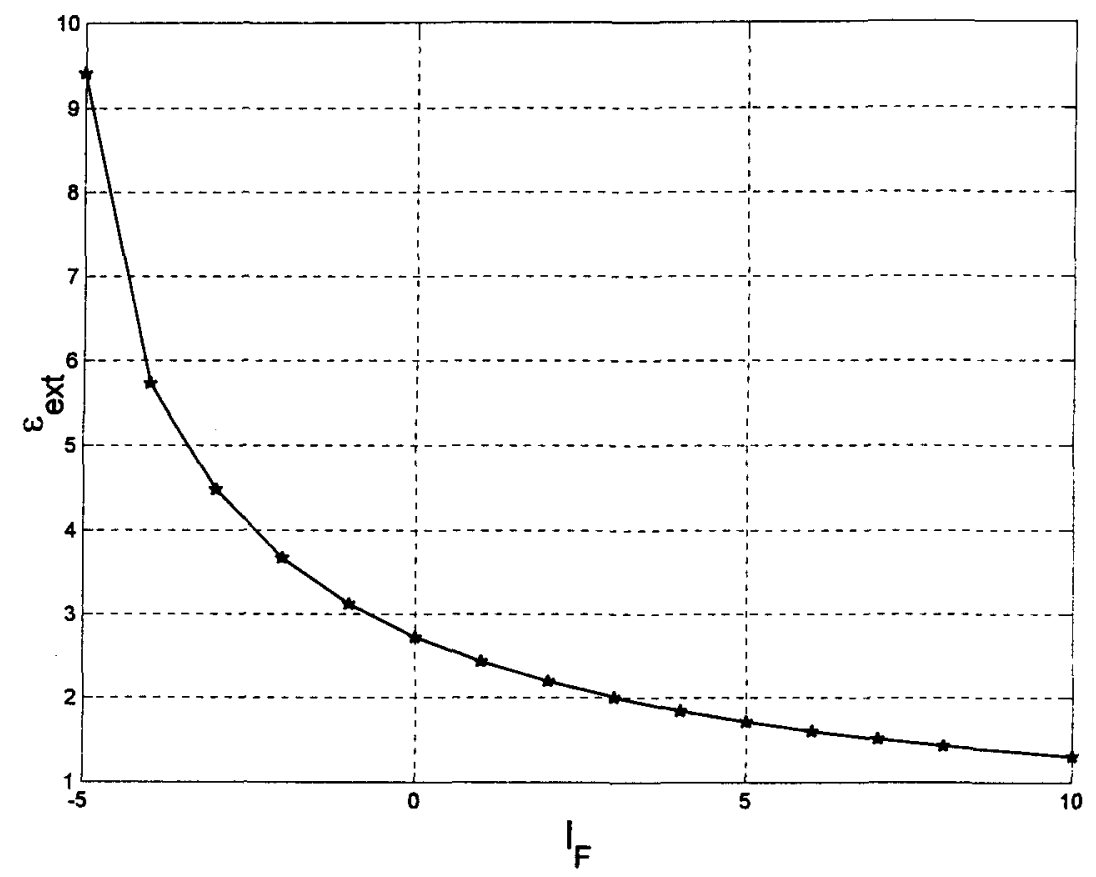

Figure 2. The value of $\epsilon$ corresponding to the extinction of the planar diffusion flame, $\epsilon_{\mathrm{ext}}$, versus $l_{\mathrm{F}}$. The values of the other parameters, kept fixed in the present study, are: $\beta=8$, $l_{O}=0, \eta_{\mathrm{s}}=0, \Upsilon=0$ and $\alpha_{\mathrm{h}}=0.85$.

\subsection{Propagation velocity of the triple flame}

Shown in figure 3 is a plot of the propagation eigenvalue $U$ versus $\epsilon$ for five values of $l_{\mathrm{F}}$, namely, $l_{\mathrm{F}}=-5,-3,0,5$ and 10 . For each value of $l_{\mathrm{F}}, U$ has been normalized by the corresponding planar flame speed, obtained numerically. Common to all cases, is the existence of a range of $\epsilon$ with negative flame speeds, as found in previous studies in unity Lewis number situations under strain [2-4]. The upper limit of this range is, as could be checked by using figure 2, the value $\epsilon_{\mathrm{ext}}$ of the planar diffusion flame. For illustration purposes, we have indicated the position of $\epsilon_{\mathrm{ext}}$ with downward arrows on the $\epsilon$-axis for $l_{\mathrm{F}}=-5,0$ and 5 .

Another important observation, is the change in the behaviour of $U$ versus $\epsilon$ as $l_{\mathrm{F}}$ is varied. The curves decrease monotonically with $\epsilon$ as long as $l_{\mathrm{F}}$ is above a critical negative value. When $l_{\mathrm{F}}$ is sufficiently negative (i.e. $L e_{\mathrm{F}}$ sufficiently below one), an important overshoot in the value of $U$ above one is observed, as seen for $l_{\mathrm{F}}=-5$. The initial increase of $U$ and its overshoot above one in such (negative $l_{\mathrm{F}}$ ) cases are essentially attributed to the rise in the flame temperature above one, and thus to the intensification of the reaction at the premixed front of the triple flame. The physical mechanisms leading to this intensification are the same as those, well known, encountered in studies of curved and stretched premixed flames. Obviously, an increase in the local combustion rate does not necessarily lead to higher values of $U$, since, due to flame curvature and to strain, only a part of the heat released is conducted towards the fresh mixture, in the direction of flame propagation. As a matter of fact, as we shall see more quantitatively below, zero and even large negative 


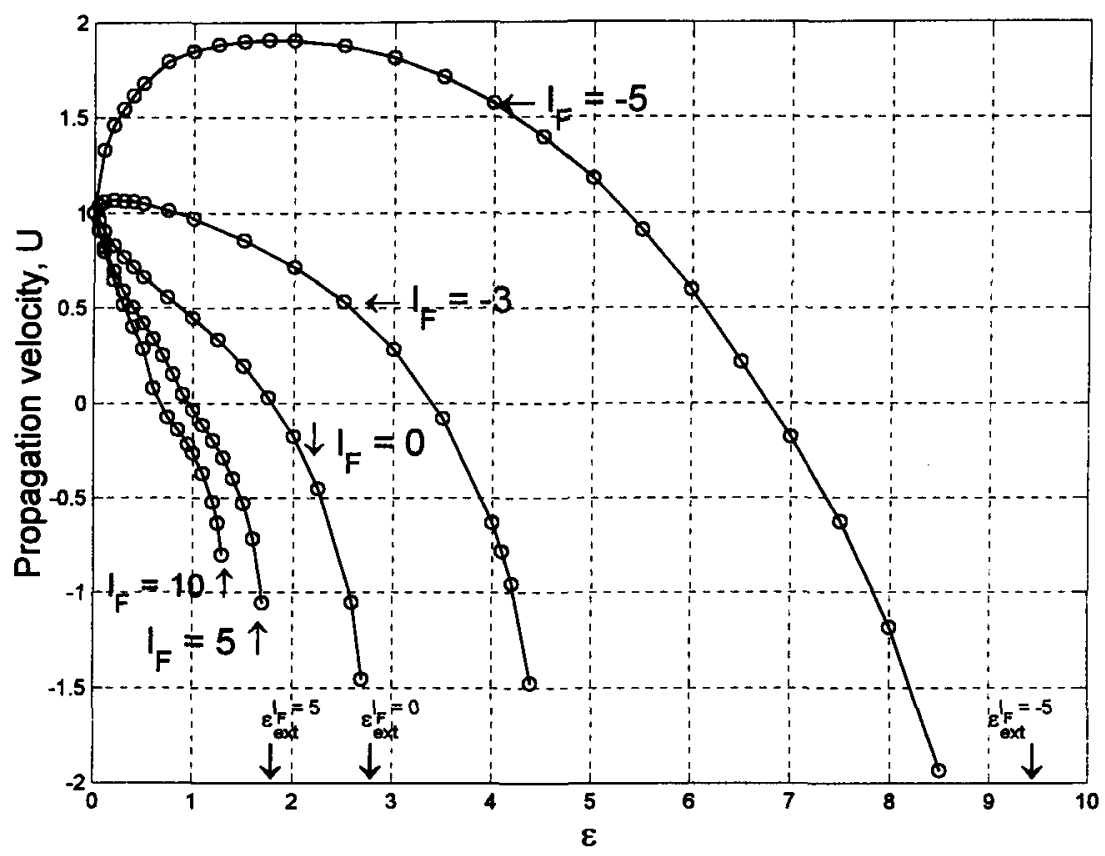

Figure 3. Propagation velocity of the triple flame, $U$, versus $\epsilon$ for five values of $l_{\mathrm{F}}: l_{\mathrm{F}}=-5$, $-3,0,5$ and 10 . For each $l_{F}, U$ has been normalized by the corresponding planar flame speed, obtained numerically. The values of $\epsilon, \epsilon_{\mathrm{ext}}$, corresponding to the extinction of the planar diffusion flame (given in figure 2) are indicated by downwards arrows on the $\epsilon$-axis for $l_{\mathrm{F}}=-5$, 0 and 5. (Values of other parameters: $\beta=8, l_{0}=0, \eta_{\mathrm{s}}=0, \Upsilon=0, \alpha_{\mathrm{h}}=0.85$.)

values of $U$ can be obtained in cases where the reaction rate is well above its corresponding level in a planar configuration.

\subsection{A comparative case for fixed $\epsilon$}

A comparison of three cases corresponding to $l_{\mathrm{F}}=-5,0$ and 5 , respectively, but to the same value of $\epsilon(\epsilon=0.5)$ is carried out in figure $4(a)$. For each of the three values of $l_{\mathrm{F}}$ under consideration, ten isocontours of the combustion rate, $\omega$, are shown. The isocontours are equidistributed between zero and the corresponding maximum value, $\omega_{\max }$, also indicated in the figure. It is seen that the quasiplanar character of the leading premixed front is progressively lost as the Lewis number is increased. Since the premixed flame is concave towards the burnt gas side, this is due to the decrease of its normal speed for increasing values of $l_{\mathrm{F}}$. Also to be noted is the shift of the leading edge and of the trailing diffusion flame from the upstream stoichiometric surface, $y=0$. In addition to this shift (towards the oxidizer side if $l_{\mathrm{O}}>l_{\mathrm{F}}$ and to the fuel side otherwise), one would expect a more important shift in the position of the leading edge in the presence of tranverse temperature gradients $\Upsilon \neq 0$, which is not included in the present numerical study.

To complement these simple observations we have plotted, in figures $4(b)$ and $(c), \Theta(x)$ and $R(x)$, respectively, defined as follows. $\Theta(x)$ is the maximum of $\theta(x, y)$ at the location $x$ as $y$ varies and $R(x)=\int_{-\infty}^{\infty} \omega(x, y) \mathrm{d} y$, that is the burning rate per unit surface parallel to the mixing layer. These two variables provide an overall and simplified description of the 
(a)
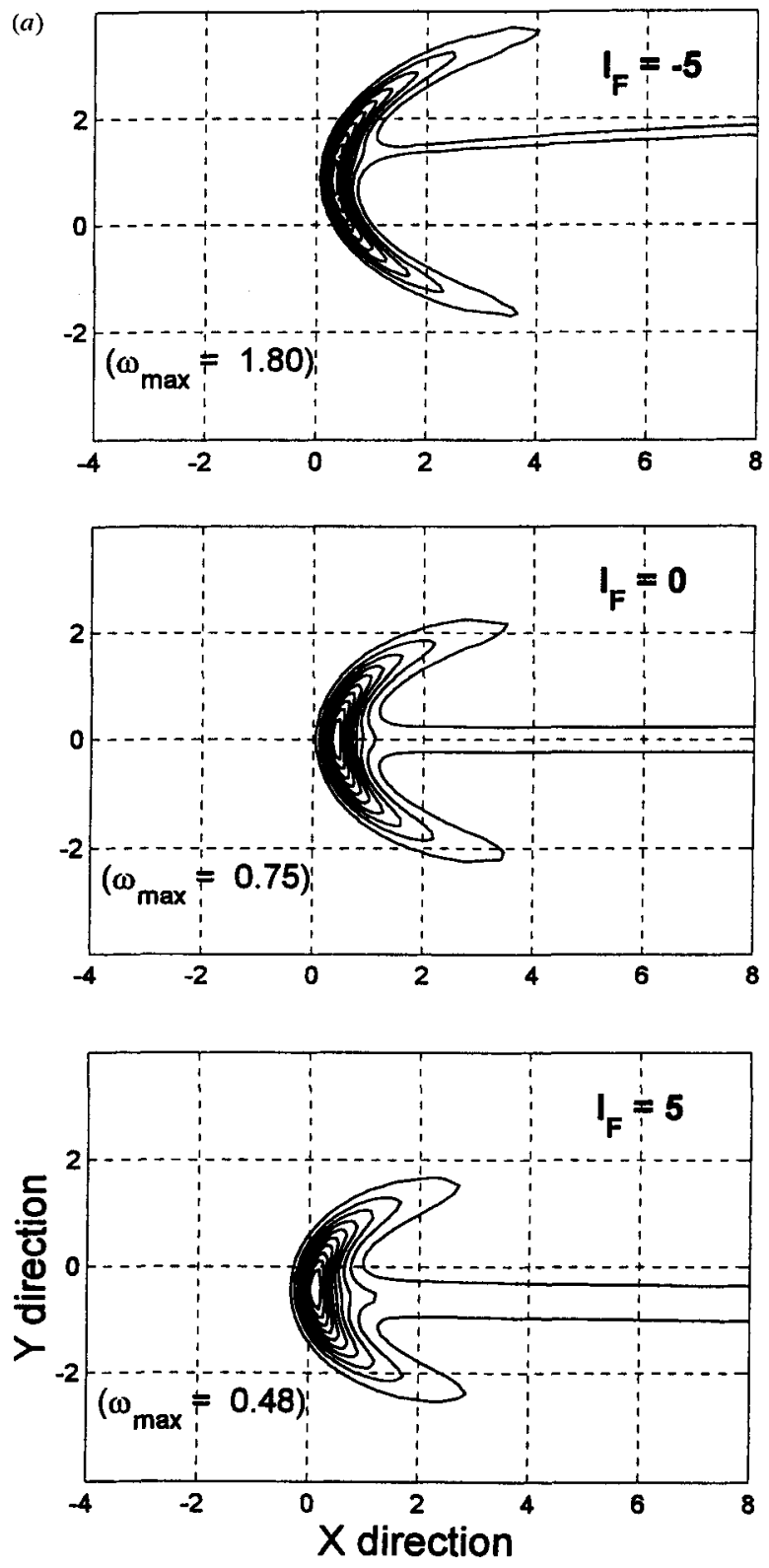

Figure 4. (a) Comparison for $\epsilon=0.5$ between three cases corresponding from top to bottom to $l_{\mathrm{F}}=-5,0$ and 5 , respectively. Each subfigure represents ten isocontours of the combustion rate, $\omega$, equidistributed between zero and the corresponding maximum value $\omega_{\max }$ (also indicated). (b) Variation of the peak temperature, $\Theta(x)$, along the mixing layer for the three cases of figure $4(a)$, corresponding to $l_{\mathrm{F}}=-5$ (full curve with stars), $l_{\mathrm{F}}=0$ (full curve) and $l_{\mathrm{F}}=5$ (full curve with squares). $\Theta(x)$ is defined as the maximum of $\theta(x, y)$ for the fixed location $x$ as $y$ varies. (c) Combustion rate per unit surface parallel to the mixing layer, $R(x)$, for the three cases of $(a)$, corresponding to $l_{\mathrm{F}}=-5$ (full curve with stars), $l_{\mathrm{F}}=0$ (full curve) and $l_{\mathrm{F}}=5$ (full curve with squares). $R(x)$ is defined by $R(x)=\int_{-\infty}^{\infty} \omega(x, y) \mathrm{d} y$. 

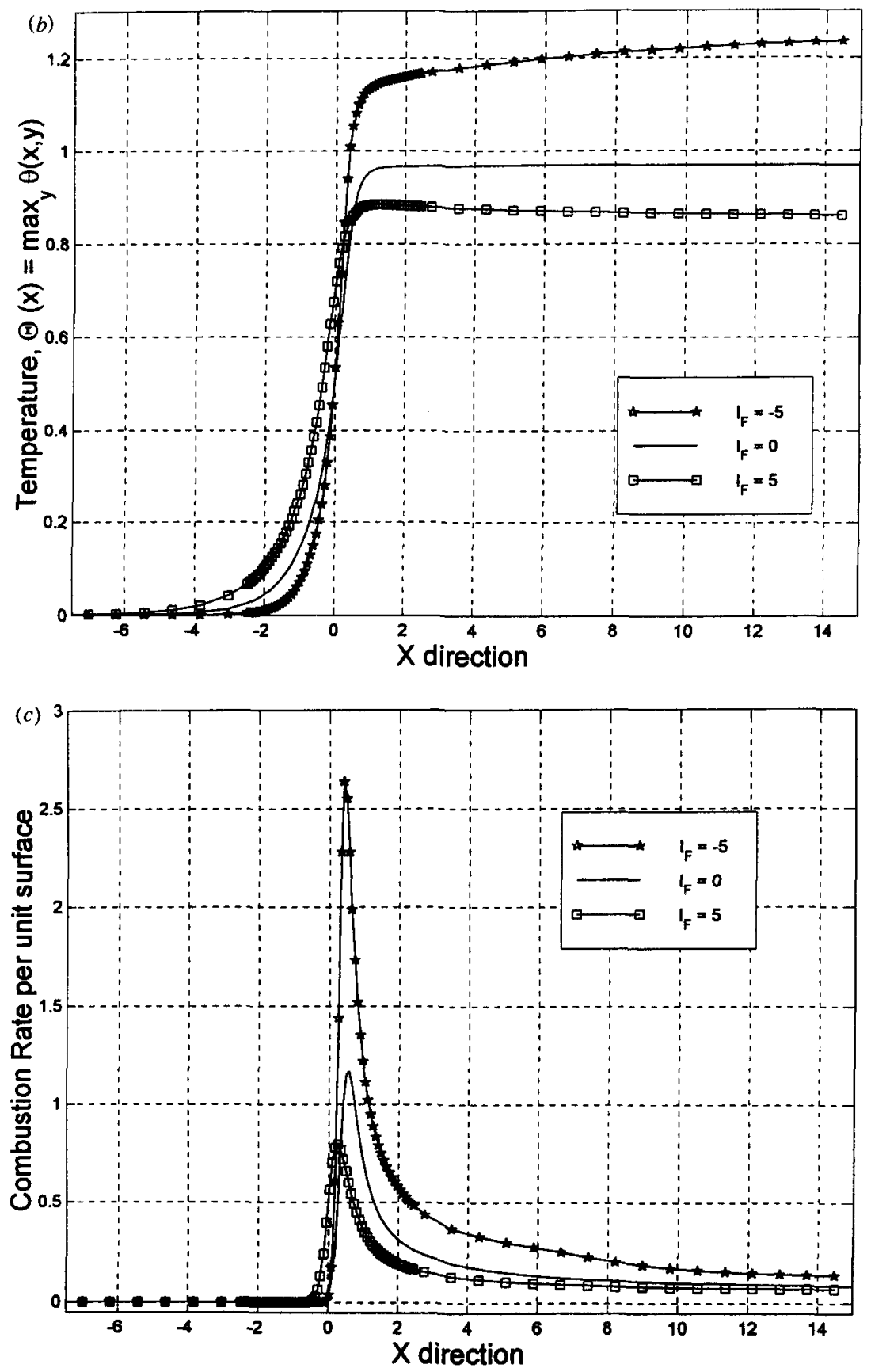

Figure 4. Continued.

combustion process in the combustible near-stoichiometric layer which, for $\beta \ll 1$, appears as a surface when viewed on a length scale of the order of, or larger than, the mixing 
(a)
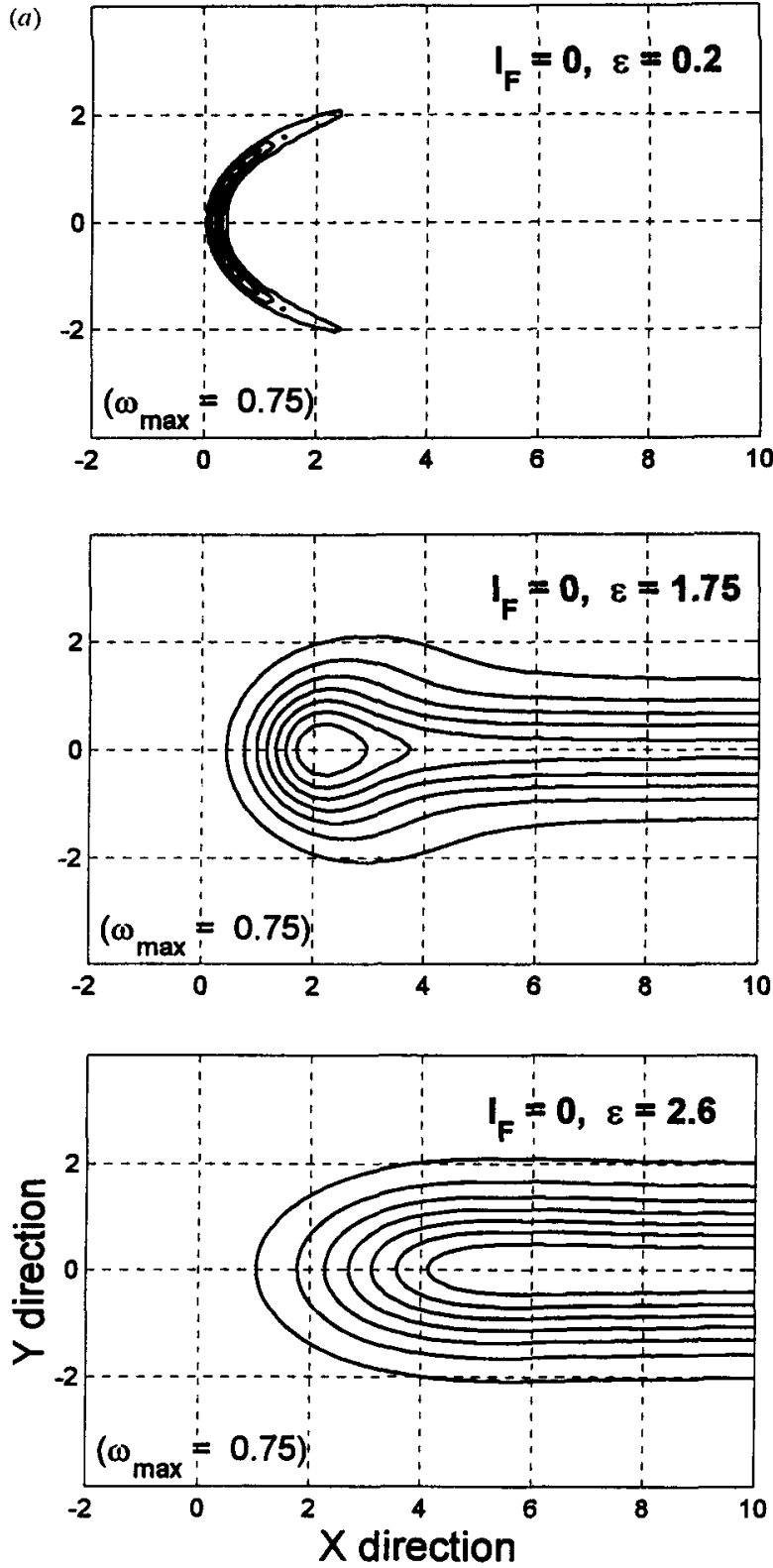

Figure 5. (a) Comparison for $l_{\mathrm{F}}=0$ between three cases corresponding from top to bottom to $\epsilon=0.2,1.75$ and 2.6 , respectively. Each subfigure represents seven isocontours of the combustion rate, $\omega$, equidistributed between zero and the corresponding maximum value $\omega_{\max }$ (also indicated). The corresponding propagation velocities (which can be extracted from figure 3 ) are equal to $0.83,0.02$ and -1.05 , respectively. (b) Peak temperature, $\Theta(x)$, and peak combustion rate $\Omega(x)$ versus $x$, corresponding frame by frame to the subfigures of $(a)$. The definition of $\Omega(x)$ is similar to that of $\Theta(x)$. (c) Peak temperature, $\Theta(x)$, and combustion rate per unit surface parallel to the mixing layer, $R(x)$ versus $x$, corresponding frame by frame to the subfigures of $(a)$. The definition of $\Theta(x)$ and $R(x)$ is as in figure 4 . 
(b)
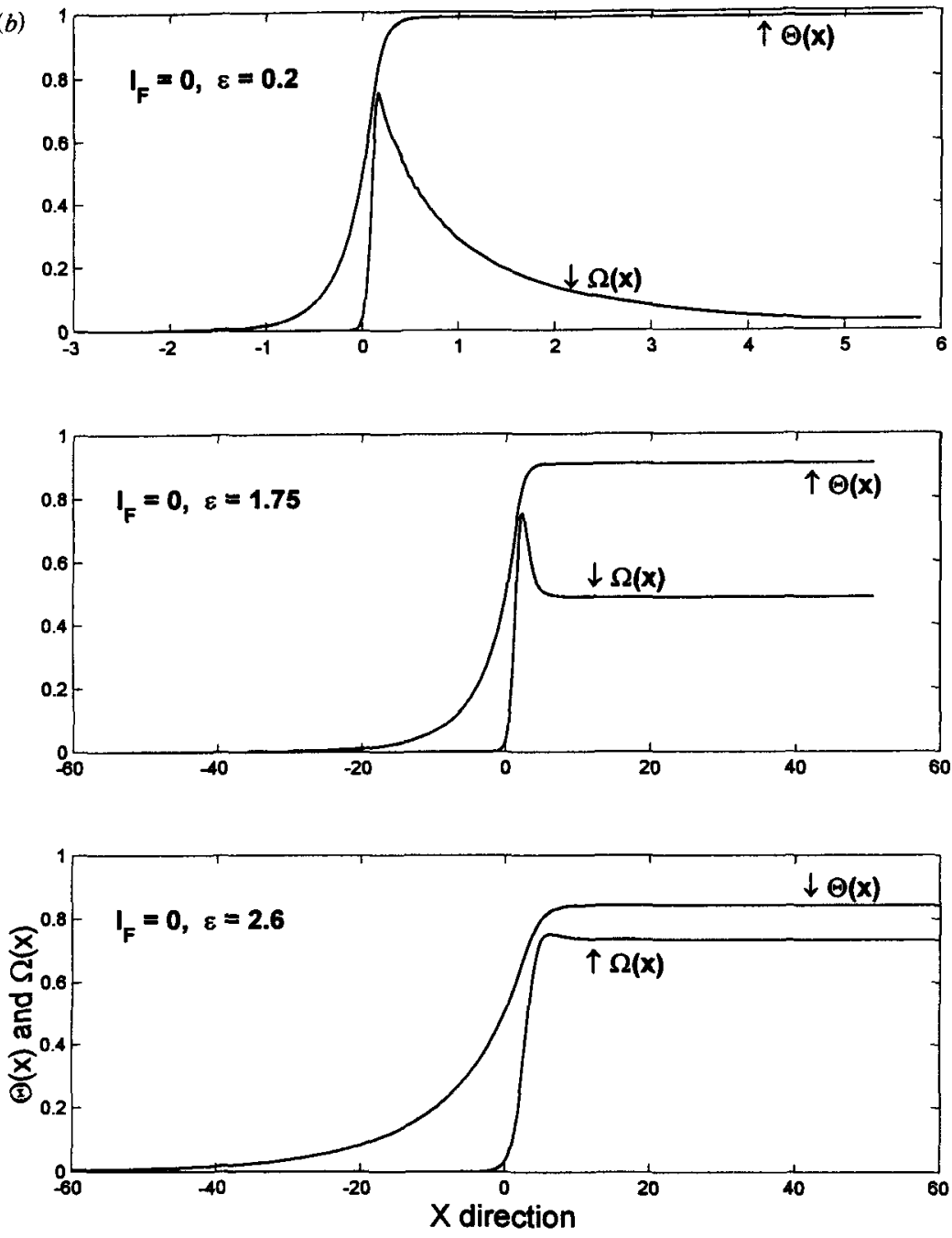

Figure 5. Continued.

layer thickness $\dagger$. We simply note in figure $4(b)$ the differences in the slopes of $\Theta(x)$ at the downstream side of the premixed front, depending on $l_{\mathrm{F}}$, varying from a positive value to a negative one as $l_{\mathrm{F}}$ is increased. No further comments will be made here in connection with figures $4(b)$ and $(c)$, since similar comments, depending on the value of $\epsilon$, will be given in the next subsection.

\subsection{Dependence on $\epsilon$ for a given Lewis number}

In this section we study the dependence on $\epsilon$, for three fixed values of $l_{\mathrm{F}}$. We begin with the results relative to $l_{\mathrm{F}}=0$, given in figures $5(a)-(c)$.

$\dagger$ Such variables can be useful in simplified one-dimensional models as in [12]. 
(c)
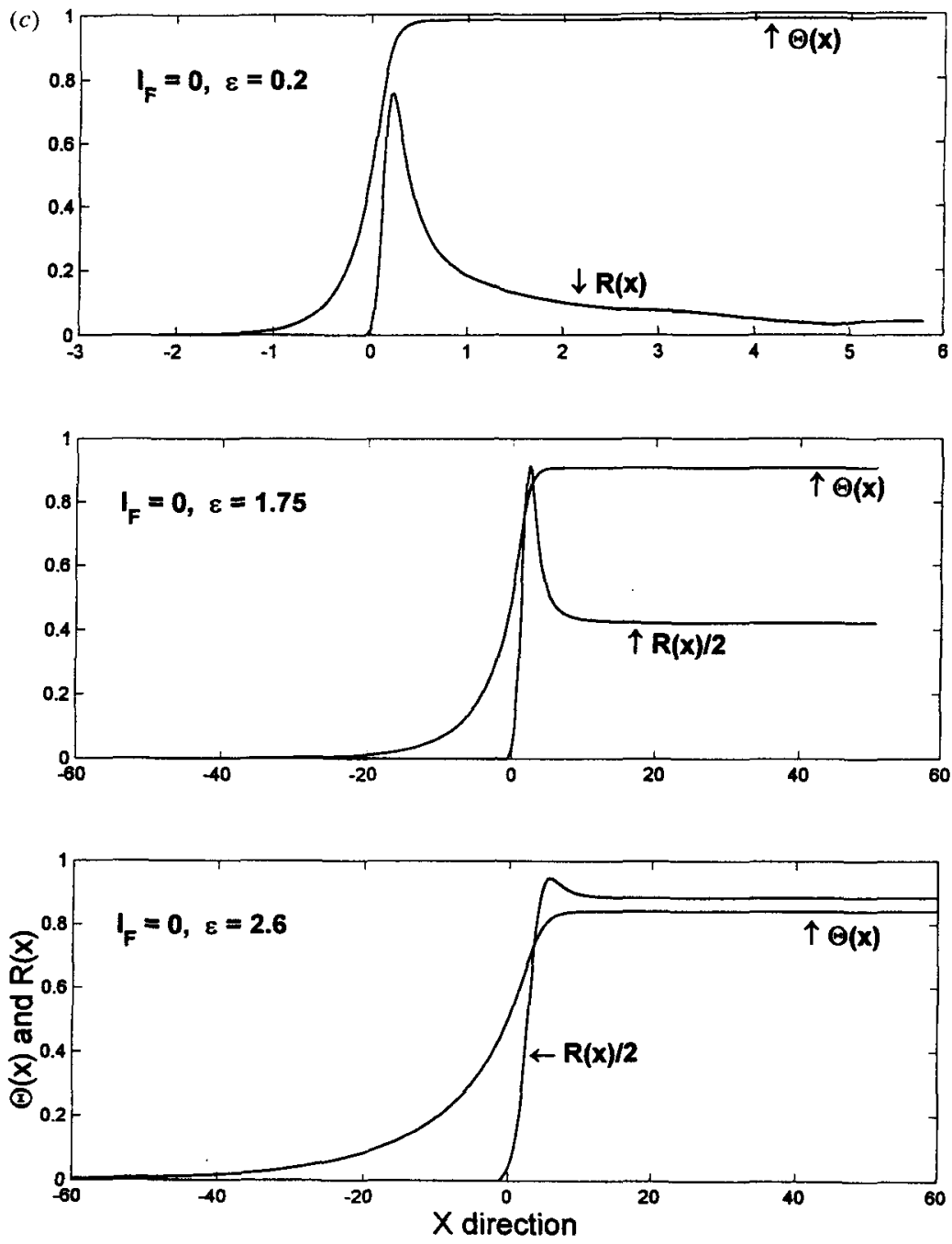

Figure 5. Continued.

Shown in figure 5(a) are three subfigures corresponding, from top to bottom, to $\epsilon=0.2$, 1.75 and 2.6, respectively. The values of $\epsilon$ are chosen such that the leading front is moderately curved, its propagation velocity is close to zero, or that it is retreating in conditions close to the extinction of the diffusion flame, $\epsilon \approx \epsilon_{\mathrm{ext}}$, from top to bottom, respectively. Each subfigure represents seven isocontours of the combustion rate, $\omega$, equidistributed between zero and the corresponding maximum value $\omega_{\max }$, also indicated. The propagation velocities corresponding to these three cases, which can be extracted from figure 3, are indicated in the captions. The triple-flame shapes observed as $\epsilon$ is varied are familiar from previous studies. Note that the maximum value of $\omega, \omega_{\max }$, remains constant, as can also be seen in figure $5(b)$, where $\Theta(x)$ (introduced above) and $\Omega(x)$ are plotted. Here $\Omega(x)$ is defined as the maximum of $\omega(x, y)$ as $y$ varies. The temperature at the hot side decreases from one, its expected maximum value for $\epsilon \rightarrow 0$, as $\epsilon$ is increased. This 
(a)
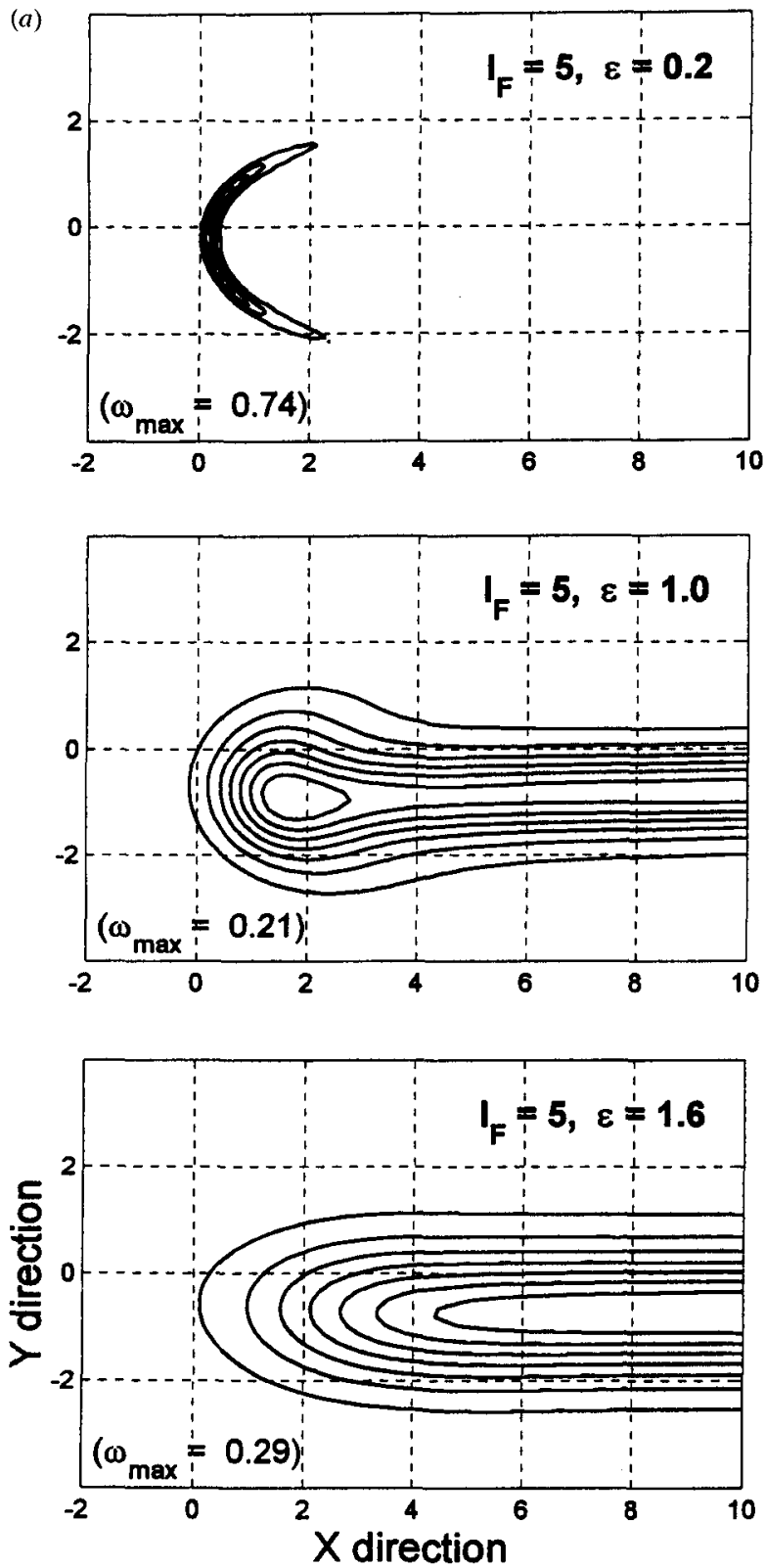

Figure 6. (a) Comparison for $l_{\mathrm{F}}=5$ between three cases corresponding from top to bottom to $\epsilon=0.2,1$ and 1.6, respectively. Each figure represents seven isocontours of the combustion rate, $\omega$, equidistributed between zero and the corresponding maximum value $\omega_{\max }$ (indicated) The corresponding propagation velocities are equal to $0.70,0.03$ and -0.72 , respectively. $(b)$ Peak temperature, $\Theta(x)$, and peak combustion rate $\Omega(x)$ versus $x$, corresponding frame by frame to the subfigures of $(a)$. 
(b)
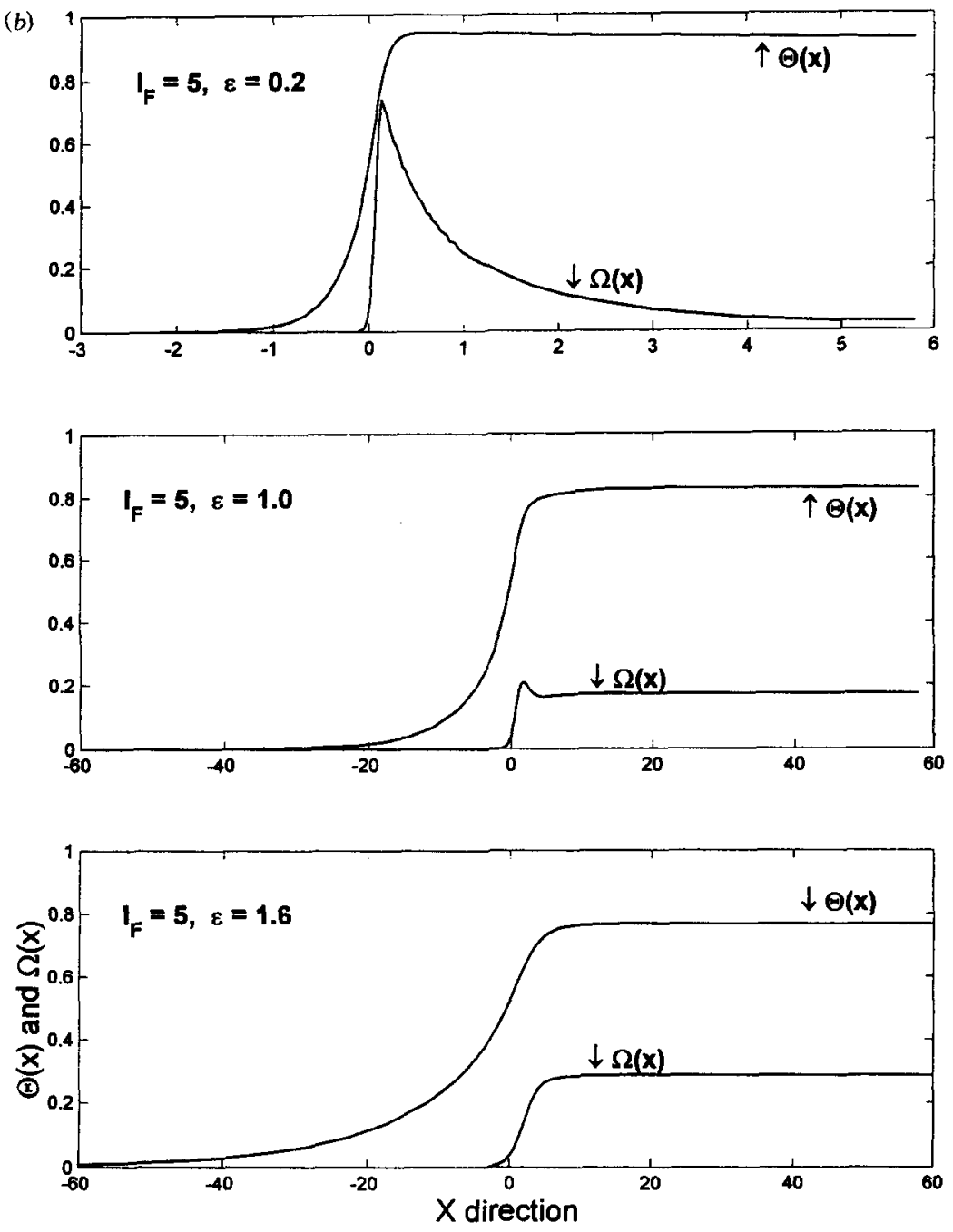

Figure 6. Continued.

decrease is not inconsistent $\dagger$ with the constancy in $\omega_{\max }$ mentioned except when $\theta$ falls by a critical amount, of order $\beta^{-1}$, below unity. In that case the reaction is quenched everywhere and both the temperature and the combustion rate fall towards their frozen values. This will happen if $\epsilon$ is increased above $\epsilon_{\text {ext }}$. Finally, figure $5(c)$ is similar to figure $5(b)$, except that the global rate $R(x)$ defined above is used instead of $\omega(x)$.

Differential-diffusion effects could be appreciated by comparing the case just presented, corresponding to $l_{\mathrm{F}}=0$, with two cases represented in figures 6 and 7 , and corresponding

$\dagger$ Indeed for $L e_{\mathrm{F}}=L e_{\mathrm{O}}=1, y_{\mathrm{O}}+\theta$ and $y_{\mathrm{F}}+\theta$ are conserved scalars which are easily determined. More precisely, $y_{0}+\theta=1+\operatorname{erf}(y / \beta)$ and $y_{\mathrm{F}}+\theta=1-\operatorname{erf}(y / \beta)$, for $\Upsilon=0$. Then, $\eta_{\mathrm{s}}$ being taken equal to zero, one obtains at the symmetry axis, $y=0$, the relations $y_{0}=1-\theta$ and $y_{\mathrm{F}}=1-\theta$, so that, $w(x, y=0)$ is a function of $\theta$ alone. Consequently, the spatial maximum of $\omega$ is equal to the maximum of the function $\omega(\theta)$, which corresponds to a given fixed value, $\theta^{*}$, independent of the problem. This is so unless $\theta$ falls everywhere below $\theta^{*}$. 
(a)
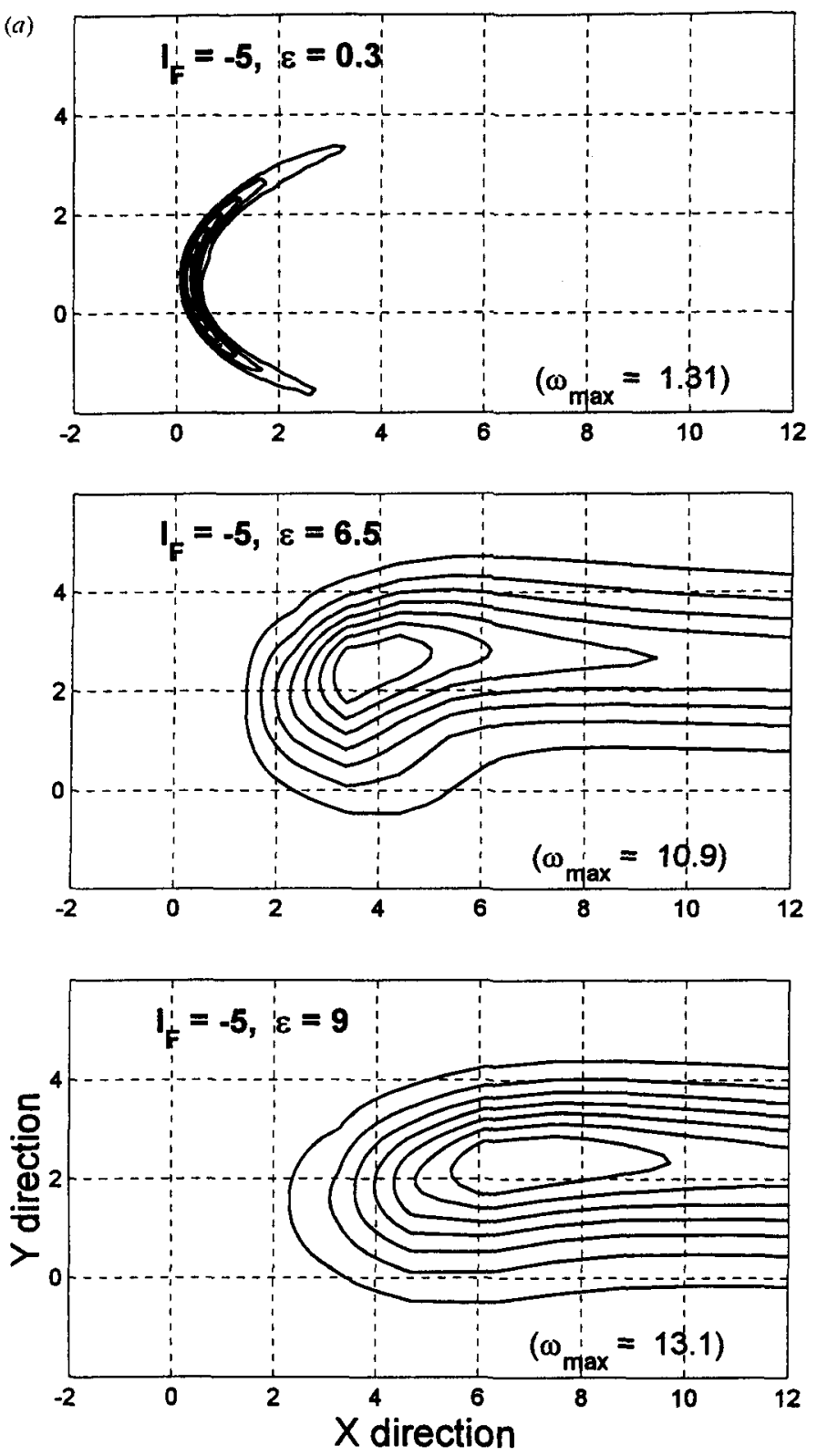

Figure 7. (a) Comparison for $l_{\mathrm{F}}=-5$ between three cases corresponding from top to bottom to $\epsilon=0.3,6.5$ and 9 , respectively. Each subfigure represents seven isocontours of the combustion rate, $\omega$, equidistributed between zero and the corresponding maximum value $\omega_{\max }$ (indicated). The corresponding propagation velocities are equal to $1.54,0.20$ and -3.04 , respectively. $(b)$ Peak temperature, $\Theta(x)$, and peak combustion rate $\Omega(x)$ versus $x$, corresponding frame by frame to the cases of $(a)$.

to $l_{\mathrm{F}}=5$ and $l_{\mathrm{F}}=-5$, respectively. The choice of the values of $\epsilon$, and the notations, demonstrated in the captions, are similar to those introduced above and need not be repeated. 

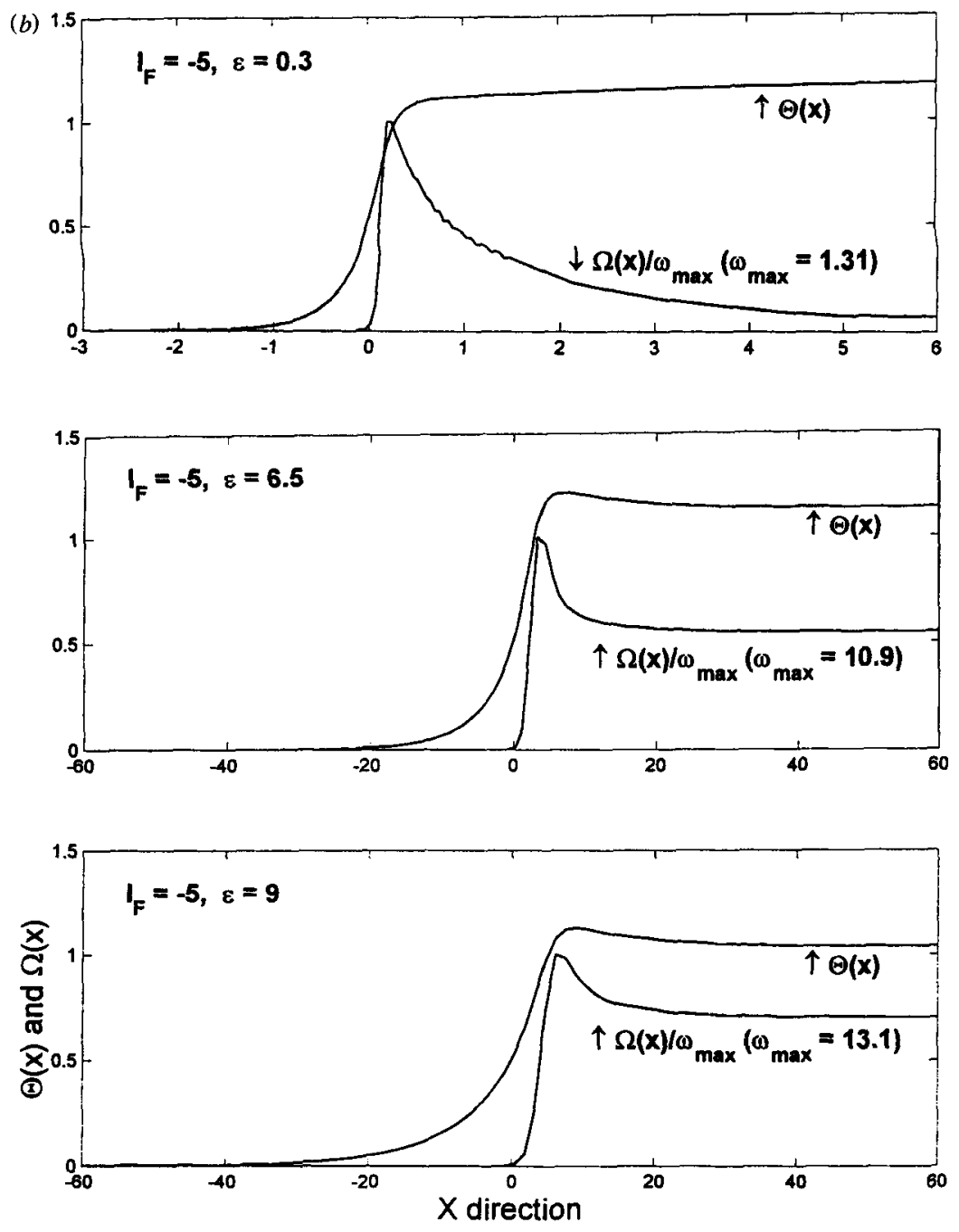

Figure 7. Continued.

It can be noted that, irrespective of $l_{F}$, the evolution of the shape of the triple flames as their propagation velocities decrease is roughly the same, despite their shift towards the fuel side for $l_{\mathrm{F}}>0$ and to the oxidizer side for $l_{\mathrm{F}}<0$. However, important differences are observed with respect to the profiles in flame temperature behind the premixed front, which depend on $\epsilon$. For example, as seen in figure $6(b)$ for $l_{\mathrm{F}}=5$, the flame temperature tends by decreasing values to its asymptotic value, corresponding to the planar diffusion flame, as $x \rightarrow \infty$, when $\epsilon$ is small; for larger $\epsilon$, it increases towards its asymptotic value. The opposite trend is observed in figure $7(b)$, for $l_{\mathrm{F}}=-5$. These observations are explained as follows.

In the limit $\epsilon \rightarrow 0$, the leading premixed front tends to be locally planar, and its burning temperature at the stoichiometric location tends to one irrespective of the value of the Lewis number. In contrast, in the same limit, the diffusion flame far downstream, which corresponds to the Burke-Schumann one-dimensional planar diffusion flame, has a burning 
temperature and position both depending on the Lewis number. To be more explicit, the position of the Burke-Schumann diffusion flame, say $y_{\mathrm{f}}$, and its burning temperature, say $\theta_{\mathfrak{f}}$, can be expressed in our notation by

$$
\theta_{\mathrm{f}}=1-\frac{l_{\mathrm{F}}}{4 \beta} \quad \text { and } \quad y_{\mathrm{f}}=-\frac{\sqrt{\pi}}{8} l_{\mathrm{F}}
$$

if $\beta \gg 1$ and, as in the numerical study, $\eta_{\mathrm{s}}=0, \Upsilon=0$ and $L e_{\mathrm{O}}=1$ are adopted $\dagger$. This difference in the burning temperature at the leading front and at the diffusion tail explains the influence of $l_{\mathrm{F}}$ on the slope of $\theta_{\max }$ for $\xi>0$, when $\epsilon$ is small. As $\epsilon$ is increased, the temperature of the diffusion tail decreases with $\epsilon$ (see figures $5(b), 6(b)$ and $7(b)$ ), while that of the leading front can increase due to flame curvature if $l_{\mathrm{F}}<0$ (figure $7(b)$ ). It is interesting to note that even negative values of $U$ are obtained, for $\epsilon$ close to $\epsilon_{\text {ext }}$, with flame temperatures and combustion rates well above their values in the planar case (figure $7(b)$ ).

\section{Quick reference to main results}

This section provides a quick reference to the main results, and could be read directly after section 2, where the scaling which leads to the governing equations (4), (5) and (7) is detailed. In particular, we recall that the reference length and speed have been taken as $L_{\text {ref }}=L / \beta$ and $U_{\text {ref }}=S_{\mathrm{L}}^{0}$, where $L=\sqrt{2 D_{\mathrm{T}} / a}$ is the mixing layer thickness, and $\beta$ and $S_{\mathrm{L}}^{0}$ are the Zeldovich number and the planar laminar flame speed corresponding to the stoichiometric conditions in the frozen mixture. Of particular importance to the present discussion are the non-dimensional parameters $\epsilon, \eta_{\mathrm{s}}$ and $\Upsilon$. The first one, defined by $\epsilon=l_{\mathrm{F}}^{0} / L_{\mathrm{ref}}$, represents the (planar) flame thickness $l_{\mathrm{F}}^{0}=D_{\mathrm{T}} / S_{\mathrm{L}}^{0}$ relative to the reference length, thus small values of $\epsilon$ correspond to thin flames or large Damköhler numbers. The second and third, defined by (6), represent the non-dimensional location of the upstream stoichiometric surface $\ddagger$ and the non-dimensional difference in the feed temperatures of the fuel and oxidizer streams, respectively. Actually, the parameters $\eta_{\mathrm{s}}$ and $\Upsilon$ appear in the analytical results in the form of two simple combinations, given in (44), namely

$$
\gamma=\frac{2 \exp \left(-\eta_{s}^{2}\right)}{\sqrt{\pi}\left(1-\operatorname{erf}\left(\eta_{s}\right)^{2}\right)} \quad \text { and } \quad \Gamma=\frac{1+\operatorname{erf}\left(\eta_{s}\right)}{2}(1-\Upsilon)
$$

For $\epsilon \ll 1$, the results are expressed as expansions in terms of $\epsilon$. In particular, the propagation velocity (the main quantity to be determined) is written as $U=U_{0}+\epsilon U_{1}$.

To leading order, $U$ is found to depend on the single parameter $\Gamma, U_{0}=U_{0}(\Gamma)$. This dependence is given by (45), a simple expression which shows that $U_{0}$ is larger than or

$\dagger$ Equations (57) can be obtained as follows. The governing equations for the underlying one-dimensional diffusion flame are given by (4) in which derivatives with respect to $x$ are discarded. The corresponding boundary conditions for $|y| \rightarrow \infty$ are obtained from (5). For $L e_{O}=1$ and $\Upsilon=0$, they are given by $\theta=y_{0}=y_{\mathrm{F}}-2\left(1-\operatorname{erf}\left(\eta_{\mathrm{s}} L e_{\mathrm{F}}^{1 / 2}\right)\right)^{-1}=0$ at $y=-\infty$, and $\theta=y_{\mathrm{F}}=y_{0}-2\left(1+\operatorname{erf}\left(\eta_{\mathrm{s}}\right)\right)^{-1}=0$ at $y=\infty$. Then, the conserved scalar $y_{\mathrm{O}}+\theta$ is found to be equal to $\left(1+\operatorname{erf}\left(\eta_{\mathrm{s}}+y / \beta\right)\right)\left(1+\operatorname{erf}\left(\eta_{\mathrm{s}}\right)\right)^{-1}$, and hence the flame temperature $\theta_{\mathrm{f}}$ is given, in the limit of infinitely fast chemistry, by $\theta_{\mathrm{f}}=\left(1+\operatorname{erf}\left(\eta_{\mathrm{s}}+y_{\mathrm{f}} / \beta\right)\right)\left(1+\operatorname{erf}\left(\eta_{\mathrm{s}}\right)\right)^{-1}$. where $y_{f}$ is the position of the reaction sheet, separating the oxygen from the fuel. To determine $y_{f}, y_{0}$ and $y_{F}$ are found at both sides of the reaction sheet by solving chemistry-free equations and using the boundary conditions at $|y|=\infty$, just given, along with $y_{\mathrm{F}}\left(y_{f}\right)=y_{O}\left(y_{f}\right)=0$. Then, the jump condition $\left(\mathrm{d} y_{\mathrm{F}} / \mathrm{d} y\right)\left(y_{\mathrm{f}}^{-}\right)=-L e_{\mathrm{F}}\left(\mathrm{d} y_{\mathrm{O}} / \mathrm{d} y\right)\left(y_{\mathrm{f}}^{+}\right)$yields the relation $\left(1+\operatorname{erf}\left(\eta_{\mathrm{s}}\right)\right)^{-1}\left(1-\operatorname{erf}\left(\eta_{\mathrm{s}}+y_{\mathrm{f}} / \beta\right)\right)^{-1} \exp \left(-\left(\eta_{\mathrm{s}}+y_{\mathrm{f}} / \beta\right)^{2}\right)=$ $L e_{\mathrm{F}}^{-1 / 2}\left(1-\operatorname{erf}\left(\eta_{\mathrm{s}} L e_{\mathrm{F}}^{1 / 2}\right)\right)^{-1}\left(1+\operatorname{erf}\left(\left(\eta_{\mathrm{s}}+y_{\mathrm{f}} / \beta\right) L e_{\mathrm{F}}^{1 / 2}\right)\right)^{-1} \exp \left(-\left(\eta_{\mathrm{s}}+y_{\mathrm{f}} / \beta\right)^{2} L e_{\mathrm{F}}\right)$. This relation, along with the one expressing $\theta_{\mathrm{f}}$ is terms of $y_{\mathrm{f}}$ given above, determine, in an implicit form, the position and temperature of the flame. In the particular case $\eta_{\mathrm{s}}=0$, these relations simplify, for $\beta \gg 1$ and $l_{\mathrm{F}}=\beta($ Le $\mathrm{F}-1)=\mathrm{O}(1)$, to the explicit form (57).

$\ddagger$ See the footnote to equation (6). 
equal to one (i.e. the stoichiometric planar flame value), accounting in particular for unequal feed temperatures. Also there is a shift in the location of the leading edge of the flame away from the upstream stoichiometric surface, given to leading order by $y^{*}$ determined in (46).

To compute the next approximation, $U_{1}$ can be evaluated from (53) where it is seen to depend on $\Gamma, \gamma$, and on the reduced Lewis numbers $l_{\mathrm{F}}=\beta\left(L e_{\mathrm{F}}-1\right)$ and $l_{\mathrm{O}}=\beta\left(L e_{\mathrm{O}}-1\right)$.

Similarly, the local normal flame speed to leading order, $S_{0}(y)$, is given by (42), which also determines the flame shape in a first approximation, $f_{0}^{\prime}$. Then, two terms expansions are available for the normal speed, $S(y)$, and for the flame temperature, $\theta_{\mathrm{Fl}}(y)$, from (54) and (55).

For illustration, consider the case corresponding to $\eta_{s}=\Upsilon=0$ (i.e. the frozen stoichiometric surface is at the centre of the mixing layer and the feed temperatures are equal). Then $\Gamma=\frac{1}{2}, \gamma=2 / \sqrt{\pi}, y^{*}=0$ (from equation (46)) and the propagation speed calculated from (45) and (53) is given by

$$
U=1-\left(1+\frac{1}{4}\left(l_{\mathrm{F}}+l_{\mathrm{O}}\right)\right) \sqrt{\frac{2}{\pi}} \epsilon .
$$

Finally, a synthetic presentation of the numerical results describing the propagation velocity of the triple flame for values of $\epsilon$ which are not necessarily small is given in figure 3 for different values of the fuel Lewis number and a fixed set of the other parameters (see captions).

\section{Conclusion}

In this study, triple-flame propagation in a strained reactive mixing layer with possibly unequal feed temperatures and non-unity Lewis numbers has been considered. The problem has been formulated within the framework of a constant-density model and an overall Arrhenius reaction. In the formulation, an important non-dimensional parameter is $\epsilon$, the ratio of the thickness of the planar stoichiometric flame to the characteristic size of the flame-front region. Numerical results describing, for different fixed values of the fuel Lewis number and growing values of $\epsilon$, the variation of the propagation velocity from positive to negative and of the front from ignition front to extinction front of the strained diffusion flame have been presented.

Special attention has been devoted to the distinguished limit $\beta \rightarrow \infty$ with $\epsilon \sim 1$. In this limit, a simplified formulation has been derived, and solved analytically for $\epsilon \ll 1$. In particular, simple expressions determining the propagation speed of the triple flame, the local burning rate and the flame shape have been obtained.

\section{Acknowledgment}

The present study was supported by the Commission of the European Communities within the framework of the programme 'Gravity Dependent Phenomena in Combustion' and postdoctoral grant no ERBFMBICT961503. 